\title{
Stochastic analysis of the impact of freestream conditions on the aerodynamics of a rectangular 5:1 cylinder
}

\author{
A. Mariotti ${ }^{\text {a,* }}$, M.V. Salvetti ${ }^{a}$, P. Shoeibi Omrani ${ }^{\text {b }}$, J.A.S. Witteveen ${ }^{\mathrm{c}, 1}$ \\ a Dipartimento di Ingegneria Civile e Industriale, Università di Pisa, Via G. Caruso 8, 56122 Pisa, Italia \\ ${ }^{\mathrm{b}}$ Heat Transfer and Fluid Dynamics Department, Netherlands Organization for Applied Scientific Research (TNO), Stieltjesweg 1, 2628CK Delft, \\ The Netherlands \\ ' Scientific Computing Group, Center for Mathematics and Computer Science (CWI), Science Park 123, 1098XG Amsterdam, The Netherlands
}

\section{A R T I C L E I N F O}

\section{Article history:}

Received 26 September 2015

Revised 2 June 2016

Accepted 7 June 2016

Available online 7 June 2016

Keywords:

Uncertainty quantification

Stochastic collocation method

BARC benchmark

Bluff-body aerodynamics

\begin{abstract}
A B S T R A C T
Uncertainty plays a significant role in the Benchmark on the Aerodynamics of a Rectangular Cylinder (BARC) with a chord-to-depth ratio of 5 . In particular, besides modeling and numerical errors, in numerical simulations it is difficult to exactly reproduce the experimental conditions due to uncertainties in the set-up parameters, which sometimes cannot be exactly controlled or characterized. In this study, the impact of the uncertainties in the inflow conditions of the BARC configuration is investigated by using probabilistic methods and two-dimensional URANS simulations. The following uncertain set-up parameters are investigated: the angle of incidence, the freestream longitudinal turbulence intensity and the freestream turbulence length scale. The stochastic collocation method is employed to perform the probabilistic propagation of the uncertainty in the three set-up parameters. This results in 25 URANS simulations based on the Smolyak sparse grid extension of the level-2 Clenshaw-Curtis quadrature points. The discretization error is estimated by repeating the same analysis on different grid sizes. Similarly, the effect of turbulence modeling is appraised by carrying out the uncertainty quantification for the Reynolds stress and the SST $k-\omega$ models. Finally, the results obtained for different assumed probability density functions of the set-up parameters are compared. The propagation of the considered uncertainties does not explain alone the dispersion of the BARC experimental data. For certain quantities of interest, the effect of turbulence modeling is more important than the impact of the uncertainties in inflow conditions. The sensitivity to the considered uncertainties also varies with the turbulence model, with a larger variability of the results obtained with the Reynolds stress model. The inflow turbulence length scale is in all cases the least important parameter.
\end{abstract}

(c) 2016 Elsevier Ltd. All rights reserved.

\section{Introduction}

The international Benchmark on the Aerodynamics of a Rectangular 5:1 Cylinder, BARC, (see [2]) was launched in 2008 and it was focused on the flow around a fixed rectangular cylinder with chord-to-depth ratio equal to 5 and infinite spanwise size. The considered flow configuration is of practical interest because many civil and industrial structures are characterized by rectangular cross sections (e.g. tall buildings, towers and bridges) and, despite the simple geometry, it contains most of the difficulties also found in realistic wind engineering problems. The 5:1 aspect ratio

\footnotetext{
* Corresponding author.

E-mail addresses: alessandro.mariotti@for.unipi.it (A. Mariotti)

mv.salvetti@ing.unipi.it (M.V. Salvetti), pejman.shoeibiomrani@tno.nl (P. Shoeibi Omrani).

1 Died in August 2015
}

is characterized by shear-layers detaching at the upstream cylinder corners and reattaching on the cylinder side rather close the downstream corners. This leads to a complex flow dynamics on the cylinder side, which adds to the vortex shedding from the rear corners and to the complex unsteady dynamics of the wake (see e.g. $[4,5])$. BARC is a blind test, without any reference observation (experimental measurements) or prediction (numerical simulations). Indeed, one of the aims of the benchmark is to assess the consistency of experimental data obtained in different wind tunnels and of numerical simulations carried out with different numerical methods and turbulence models. Clearly, the comparison between experimental and numerical predictions of the flow and of the aerodynamics loads is also an important objective of the benchmark.

About 70 realizations of the BARC flow configuration, obtained in both wind tunnel experiments and numerical simulations, were reviewed by Bruno et al. [5]. A significant dispersion was observed 
both in experimental and numerical predictions of some quantities of practical interest, as the standard deviation of the lift coefficient or the distribution of mean and fluctuating pressure on the cylinder sides. Therefore, these quantities seem to be extremely sensitive to various uncertainties, which may be present in experiments and numerical simulations. The sources of uncertainty are in general different in computations and experiments. We focus here on the uncertainties in the incoming flow conditions, which may be considered as 'common' to computations and experiments. Indeed, in the experiments it is difficult to perfectly control and/or characterize some features of the incoming flow, for example the angle of incidence or the turbulence characteristics. The choice of these uncertain parameters follows the analysis in [5] and its rationale will be explained in more detail in the following. Hence, the present paper wish to give a contribution to understand whether the uncertainties in the incoming flow features have a significant impact on the flow characteristics and on the aerodynamic loads, which may explain the dispersion among the experimental data and the discrepancies between simulation and experimental results.

To this aim, sensitivity analysis and Uncertainty Quantification (UQ) are carried out using probabilistic methods. In this framework, the uncertain set-up parameters are considered as random variables having an a-priori given Probability Density Function (PDF). In the present work, the following uncertain set-up parameters are considered: the angle of incidence, the longitudinal turbulence intensity, and the turbulence length scale of the incoming flow. The remaining characteristics of the incoming flow are fixed; in particular the freestream velocity, $u_{\infty}$, and the fluid kinematic viscosity, $v$, are such that the Reynolds number, based on the cylinder depth, $D$, is equal to $\operatorname{Re}_{D}=\left(u_{\infty} D\right) / v=40000$. Variations in the Reynolds number are not considered, because it has been found that Reynolds number effects on the flow are not among the predominant ones in the range recommended for the BARC benchmark, $20000 \leq R e_{D} \leq 60000$ (see [5]).

The computational model which was used to propagate the uncertainties in the considered set-up parameters is based on the 2D Unsteady Reynolds-Averaged Navier-Stokes (URANS) equations. The choice of the URANS approach to turbulence was mainly motivated by the need of keeping the computational effort feasible. Note that URANS computations represented about the $30 \%$ of the numerical contributions reviewed in [5] and it was concluded that none of the approaches to turbulence, namely URANS, Large-Eddy Simulation (LES) and hybrid URANS/LES, seem to reduce the dispersion of the results. The choice of carrying out $2 \mathrm{D}$ simulations is consistent with that made by the other contributors to BARC running URANS simulations ([5]). Moreover, no large differences were found in a previous study ([10]) between 2D and 3D URANS solutions, and it was observed that the flow field resolved by the URANS solutions contained only limited three-dimensional flow features.

In order to obtain a continuous response surface of the flow quantities of interest in the parametric space at a reasonable computational cost, a Stochastic Collocation (SC) method (see [19]) is employed, in which the variables are interpolated in the parameter space at quadrature points through Lagrange polynomials. The Clenshaw-Curtis quadrature points are used. Different levels of these quadrature points can be defined; higher levels may be considered to correspond to a finer resolution in the parameter space. This method is extended to multiple dimensions using Smolyak sparse grids (see [16]). For three uncertain parameters, as in our case, this results, for instance, in 25 URANS simulations based on the Smolyak sparse grid extension of the level-2 Clenshaw-Curtis quadrature points. The convergence of the stochastic UQ procedure is estimated by comparing the outputs with those on the nested lower levels. The result of this procedure is a probabilistic quantification of the impact of the considered uncertainties in terms of the stochastic mean value, variance and PDF of the output quanti- ties of interest. The output quantities of interest considered in the following are the time statistics (time-average and standard deviation) of the aerodynamic loads acting on the cylinder, of the pressure and of the velocity fields. The UQ results are also compared with the ensemble statistics of the available numerical and experimental data sets [5].

The impact of spatial discretization and of turbulence modeling is also estimated by repeating the UQ and sensitivity analyses for two different grid resolutions and two different turbulence models, namely the Reynolds stress [9] and the SST $k-\omega$ [11] models.

Finally, the effect on the UQ results of the assumed PDF shape for the input uncertain parameters is investigated, by repeating the analysis for different input PDFs.

The paper is organized as follows. The simulation set-up and the numerical methodology are described in Section 2. The grid and statistic convergence is assessed in Section 3, in which the main flow features are also described. The uncertainty quantification methodology is presented in Section 4, while the definition of the text matrix and the convergence of the UQ procedure are shown in Section 5. The impact of uncertainties in the selected input parameters on the results obtained for the Reynolds stress model at grid independence is discussed in Section 6. The effect of the chosen PDF of the input parameters on the UQ results is analyzed in Section 7, while those of the turbulence model and of the grid resolution are described in Sections 8 and 9, respectively. Concluding remarks are given in Section 10.

\section{Simulation set-up and numerical methodology}

Two-dimensional incompressible URANS simulations have been carried out at Reynolds number $R e_{D}=\left(u_{\infty} D\right) / v=40000$, based on the cylinder depth, $D$, the freestream velocity, $u_{\infty}$, and the fluid kinematic viscosity, $v$. This Reynolds number is in the range recommended for the BARC benchmark $\left(20000 \leq R e_{D} \leq 60000\right)$. Moreover, it was observed in [5] that changes in Reynolds number within that range do not significantly affect the flow features.

The commercial code Fluent (see e.g. [1]) was used. Two different turbulence models are used and compared, namely the ShearStress Transport $k-\omega$ (SST $k-\omega)$ [11] and the Reynolds Stress Model (RSM)[9]. For both turbulence models, no wall functions are used and a suitable grid refinement is adopted in order to have $y^{+} \leq 1$ at the wall.

The space discretization of the URANS equations is based on finite volumes together with a second-order upwind scheme. Unsteady time advancing is chosen together with a second-order implicit time-advancing scheme. The adopted dimensionless time step is $\Delta T=\Delta t /\left(D / u_{\infty}\right)=1.6 \times 10^{-2}$. This corresponds to more than 500 time steps in each shedding cycle. It has been checked that reducing the time step by a factor three did not bring any noticeable difference in the results. The segregated PISO algorithm (Pressure-Implicit with Splitting of Operators) is chosen to couple the pressure and momentum equations (see e.g. [8]).

The origin of the chosen reference frame is located at the center of the rectangular cylinder, whose coordinates are $x / D \in[-2.5,2.5]$ and $y / D \in[-0.5,0.5]$. The computational domain is rectangular and has the following dimensions: $x / D \in[-75,125]$ and $y / D \in$ $[-75,75]$. These dimensions are the same as those used in the $x$ and $y$ directions in the LES of the BARC benchmark in [7] (also reported in [5]).

As regards the boundary conditions, at the boundaries at $x / D=$ -75 and at $y / D= \pm 75$, the modulus and the direction of the velocity as well as the turbulence intensity $I$ are specified. A pressure boundary condition is imposed at the outlet section $(x / D=125)$ and, as mentioned before, no-slip conditions are applied at the cylinder surfaces. 


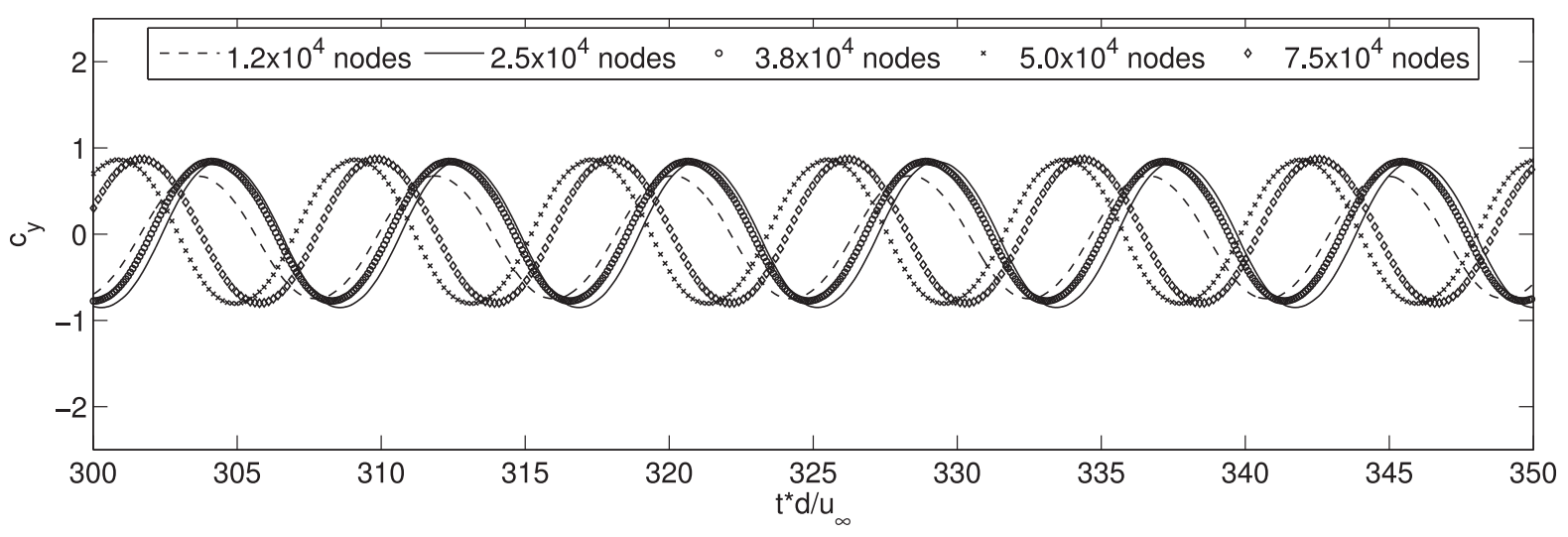

(a) Vertical-force coefficient

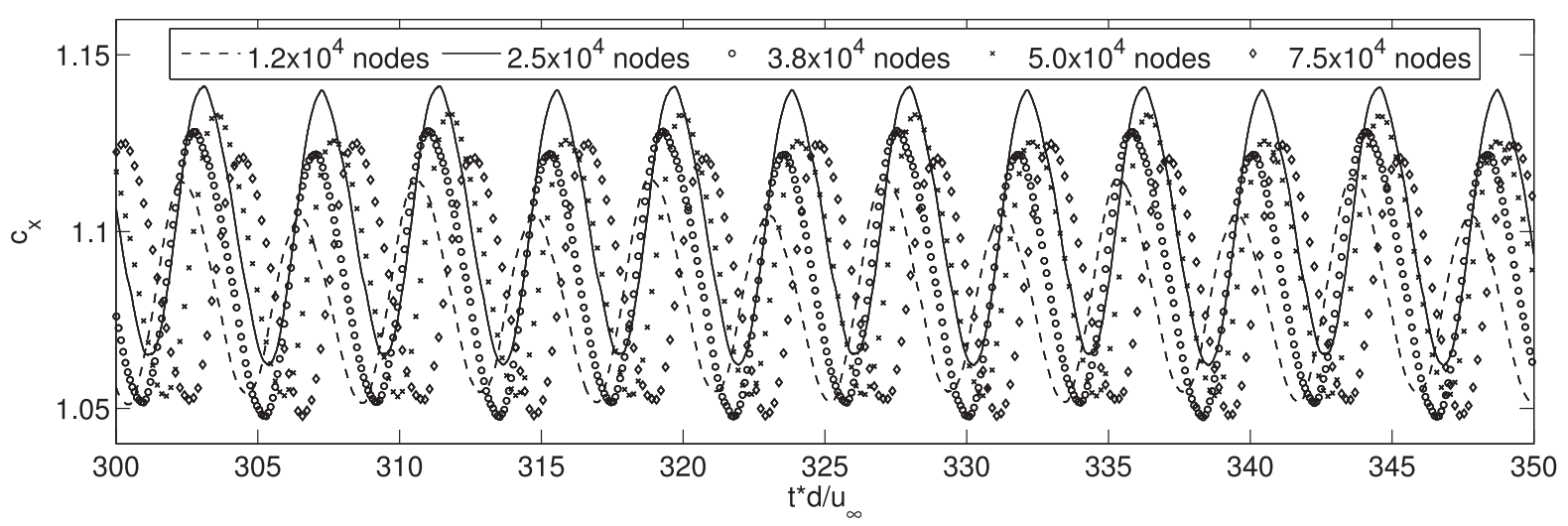

(b) Horizontal-force coefficient

Fig. 1. Time behavior of the force coefficients (RSM).

Table 1

Main grid parameters.

\begin{tabular}{llll}
\hline Grid nodes & $\overline{n_{w}} / B$ & $\overline{n^{+}}$ & $\overline{\delta_{s}} / B$ \\
\hline $1.2 \times 10^{4}$ & $1.7 \times 10^{-3}$ & $\cong 2.5$ & $2 \times 10^{-3}$ \\
$2.5 \times 10^{4}$ & $8.7 \times 10^{-4}$ & $\cong 2$ & $1 \times 10^{-3}$ \\
$3.8 \times 10^{4}$ & $5.5 \times 10^{-4}$ & $\cong 1.5$ & $6.4 \times 10^{-4}$ \\
$5.0 \times 10^{4}$ & $4.2 \times 10^{-4}$ & $\cong 1$ & $4.8 \times 10^{-4}$ \\
$7.5 \times 10^{4}$ & $3.3 \times 10^{-4}$ & $\cong 0.5$ & $3.8 \times 10^{-4}$ \\
\hline
\end{tabular}

The computational grids are unstructured and are composed of triangular elements. They are described in more detail in Section 3, together with the results of a grid sensitivity analysis carried out for the RSM.

\section{Main flow features and convergence assessment}

In this Section the convergence of time-statistics is analyzed and a grid independence study is presented for the RSM turbulence model and for the values of the input parameter equal to $\alpha=0^{\circ}, I=1.55 \%$, and $L=2.55 \mathrm{D}$. The results of these analyses can be extended to the whole range of variation of the parameters, under the assumption that the relatively small parameter variations have a small effect on the convergence of statistics.

Five different grid resolutions are considered, having $1.2 \times 10^{4}$, $2.5 \times 10^{4}, 3.8 \times 10^{4}, 5.0 \times 10^{4}$ and $7.5 \times 10^{4}$ nodes, respectively. The grids are all unstructured and made of triangular elements.
The average grid spacing on the cylinder surface, $\overline{\delta_{s}}$, is given in Table 1 ; the node distribution is not uniform, being slightly clustered near the cylinder corners. Table 1 also shows the average grid spacing normal to the cylinder surface, $\overline{n_{w}}$ and the corresponding average resolution in wall units, $\overline{n^{+}}$. All the grids are symmetric with respect to the $x$ axis.

\subsection{Aerodynamic loads}

Oscillating aerodynamic loads act on the rectangular cylinder. Force coefficients in the $x$ and $y$ directions can be defined as follows:

$c_{x}=\frac{F_{x}}{1 / 2 \rho u_{\infty}^{2} D} \quad c_{y}=\frac{F_{y}}{1 / 2 \rho u_{\infty}^{2} D}$

in which $F_{x}$ and $F_{y}$ are the resultant aerodynamic forces in the $x$ and $y$ directions respectively. The time behavior of the force coefficients is shown in Fig. 1 for all the considered grids. Note that the force coefficients in the $x$ and $y$ directions coincide with drag and lift coefficients only in the cases at $\alpha=0$.

Another quantity of interest is the frequency, $f$, of the time oscillations of the vertical-force coefficient, which is connected with the frequency of vortex shedding from the rear corners. The related Strouhal number, based on the body diameter and the freestream velocity, is defined as $S t=f D / u_{\infty}$. The Strouhal number is found to be equal to $0.112 \pm 0.004$ for all the grids. Previous simulations and BARC experiments give values of $S t$ in the range $0.105-0.12$ [5]. 
Table 2

Convergence of time statistics of the aerodynamics loads for the different grid resolutions (RSM).

\begin{tabular}{cccll}
\hline Grid nodes & Time range & $t-\operatorname{avg}\left(c_{y}\right)$ & $t-\operatorname{avg}\left(c_{x}\right)$ & $t-\operatorname{std}\left(c_{y}\right)$ \\
\hline $1.2 \times 10^{4}$ & {$[300,350]$} & -0.0450 & 1.0813 & 0.5133 \\
& {$[300,450]$} & $-\mathbf{0 . 0 4 3 2}$ & $\mathbf{1 . 0 8 1 4}$ & $\mathbf{0 . 5 1 1 6}$ \\
& {$[300,550]$} & -0.0409 & 1.0816 & 0.5111 \\
& {$[300,350]$} & -0.0054 & 1.1020 & 0.6130 \\
& & & & \\
& {$[\mathbf{3 0 0 , 4 5 0}]$} & $\mathbf{- 0 . 0 0 5 1}$ & $\mathbf{1 . 1 0 1 9}$ & $\mathbf{0 . 6 1 2 8}$ \\
& {$[300,550]$} & -0.0048 & 1.1019 & 0.6124 \\
& {$[300,350]$} & 0.0451 & 1.0875 & 0.5870 \\
& {$[\mathbf{3 0 0 , 4 5 0}]$} & $\mathbf{0 . 0 4 6 3}$ & $\mathbf{1 . 0 8 7 4}$ & $\mathbf{0 . 5 8 5 9}$ \\
& {$[300,550]$} & 0.0477 & 1.0874 & 0.5847 \\
& {$[300,350]$} & 0.0355 & 1.0926 & 0.6082 \\
& {$[\mathbf{3 0 0 , 4 5 0}]$} & $\mathbf{0 . 0 3 4 7}$ & $\mathbf{1 . 0 9 2 1}$ & $\mathbf{0 . 6 0 7 4}$ \\
$5.0 \times 10^{4}$ & {$[300,550]$} & 0.0299 & 1.0923 & 0.6041 \\
& {$[300,650]$} & 0.0275 & 1.0922 & 0.6057 \\
& {$[300,750]$} & 0.0271 & 1.0923 & 0.6051 \\
& {$[300,350]$} & 0.0438 & 1.0875 & 0.6015 \\
$7.5 \times 10^{4}$ & {$[\mathbf{3 0 0 , 4 5 0}]$} & $\mathbf{0 . 0 4 6 6}$ & $\mathbf{1 . 0 8 6 8}$ & $\mathbf{0 . 6 0 4 1}$ \\
& {$[300,550]$} & 0.0404 & 1.0869 & 0.6020 \\
\hline
\end{tabular}

\subsection{Convergence analysis of the time-statistics}

In all cases a numerical transient equal approximately to $T=$ $t * u_{\infty} / D=300$ is present, which is not considered in the computation of the time-statistics. Different time intervals between $T=300$ and $T=550$ have been considered for the analysis of the convergence of the time-statistics on all the grids. The values of $t-\operatorname{avg}\left(c_{x}\right)$ and $t-\operatorname{std}\left(c_{y}\right)$ computed over 150 non-dimensional time units and those evaluated over $250 T$ show very small differences, the maximum variation being lower than $0.5 \%$ (see Table 2 ). The value of $t-\operatorname{avg}\left(c_{y}\right)$ should be zero for these simulations carried out at zero incidence, while we obtain a value that is not exactly zero. To check whether this behavior can be due to a lack of statistical convergence, statistics have been computed for the simulation on the grid having $5.0 \times 10^{4}$ nodes also over $350 T$ and 450T. With increasing statistical sample the value of $t-\operatorname{avg}\left(c_{y}\right)$ slightly decreases but it does not seem to converge to zero. Note that values of $t-\operatorname{avg}\left(c_{y}\right)$ significantly larger than those reported in Table 2 were obtained in some of the numerical contributions to BARC (see Tab. 9 in [5]). In particular, the large values of $t-\operatorname{avg}\left(c_{y}\right)$ of the LES by [3] were probably not due a lack of convergence, since a careful check of the statistical convergence had been carried out, nor to an asymmetry of the grid. In this framework, we think that the values obtained in our simulations may be acceptable as a starting point for the following stochastic sensitivity analysis. As it will be shown in the following, the values of $t-\operatorname{avg}\left(c_{y}\right)$ in Table 2 are at least one order of magnitude smaller than the variations due to the uncertainties in the angle of incidence.

Based on the previous analysis, all the time-statistics used in the following of the work are computed over 150T, i.e. in the time interval $T \in[300,450]$. It should be noted that this time interval is much lower than the time interval required to obtain convergence for LES. This, however, is not surprising since 2D URANS simulations give a more periodic flow dynamics than LES (see e.g. [5]).

\subsection{Main flow features}

The mean velocity streamlines are reported in Fig. 2 for the grid having $5.0 \times 10^{4}$ nodes. As can be seen, the mean flow detaches from the corners of the cylinder and leads to the formation of two mean recirculation areas aside of the cylinder. Then, it separates again from the corners at the rear side.

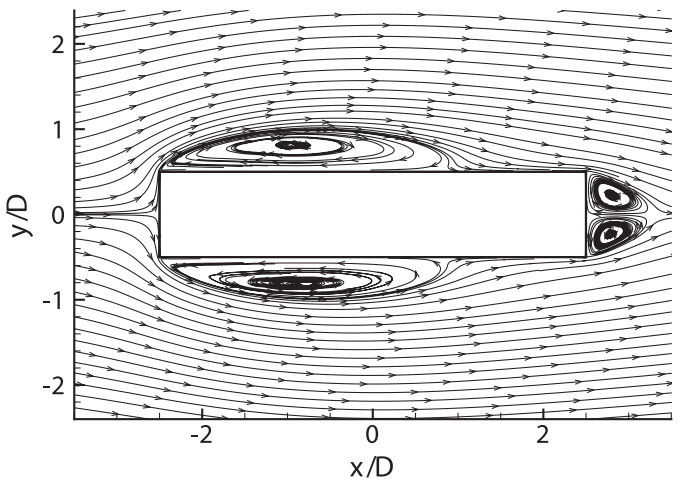

Fig. 2. Mean flow streamlines (RSM and grid having $5.0 \times 10^{4}$ nodes).

Fig. 3 (a) shows for all the grids the distributions of the pressure coefficient, defined as $C_{p}=\left(p(x, y, t)-p_{\infty}\right) /\left(1 / 2 \rho u_{\infty}^{2}\right)$, averaged in time ( $t-a v g$ in the following) and between the upper and lower half perimeters of the cylinder (side - avg in the following), while the related distributions of the side-averaged standard deviation in time ( $t-s t d$ in the following) of the pressure coefficient is reported in Fig. 3(b). As in [5], the local abscissa $s / D$ denotes the distance from the cylinder stagnation point measured along the cylinder side. Note that side-averaging of $t-\operatorname{avg}\left(C_{p}\right)$ and of $t-\operatorname{std}\left(C_{p}\right)$ has been performed to provide a more direct comparison with the results in [5]; it has been previously checked that the distributions obtained on the upper and lower half perimeters are very similar, the maximum difference being 0.024 for $t-\operatorname{avg}\left(C_{p}\right)$ and 0.057 for $t-\operatorname{std}\left(C_{p}\right)$. By comparing Fig. 3(a) and (b) to Fig. 2 it is evident that the mean pressure distribution on the cylinder side is directly related to the curvature of the mean streamlines, with a first zone of almost constant low pressure, whose length roughly corresponds to the distance from the upstream corner to the center of the main recirculation. Further downstream on the cylinder sides, the pressure increases because of the change in the curvature of the mean streamlines as the mean flow tends to reattach. Also, the maximum standard deviation of the pressure coefficient is located at the flow reattachment points aside of the model.

\subsection{Grid sensitivity}

The statistics of the aerodynamic loads are presented in Table 2 for the interval $T \in[300,450]$ obtained on the five different grids having increasing resolution. Starting from the grid having $5.0 \times 10^{4}$ nodes, the variations of $t-\operatorname{avg}\left(c_{x}\right)$ and $t-\operatorname{std}\left(c_{y}\right)$ with finer grid resolution are lower than $0.5 \%$, while those of the other parameters are even lower. Moreover, the value of the time-averaged horizontal-force coefficient, $t-\operatorname{avg}\left(c_{x}\right)$, is close to the ensemble average of all the numerical contributions to BARC, which was of $1.074(5)$, and to the experimental data of $[13,14](t-$ $\left.\operatorname{avg}\left(c_{x}\right)=1.029\right)$. The value of $t-\operatorname{std}\left(c_{y}\right)$ is also in good agreement with those obtained in URANS simulations of BARC (see Table 9 in 5). As for $t-\operatorname{avg}\left(c_{y}\right)$, similar considerations to those in Section 3.2 concerning statistical convergence can be made. The values of $t-\operatorname{avg}\left(c_{y}\right)$ do not show a monotonic behavior with grid refinement.

Fig. 3 (a) and (b) confirm that grid independency has been reached also for the pressure distribution on the cylinder surface also for the grid having $5.0 \times 10^{4}$ nodes. The maximum difference between the values obtained with the grid having $5.0 \times 10^{4}$ and those computed on the $7.5 \times 10^{4}$ grid is 0.01 for side $-\operatorname{avg}(t-$ $\left.\operatorname{avg}\left(C_{p}\right)\right)$ and 0.034 for side $-\operatorname{avg}\left(t-\operatorname{std}\left(C_{p}\right)\right)$.

Based on the previous analysis, the grid having $5.0 \times 10^{4}$ nodes will thus be used in the uncertainty quantification procedure. The results on the other grids can be used to estimate the impact of 


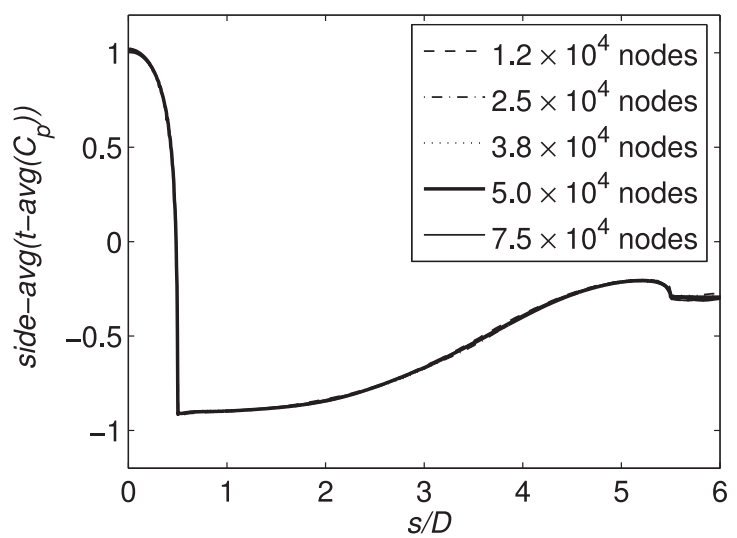

(a)

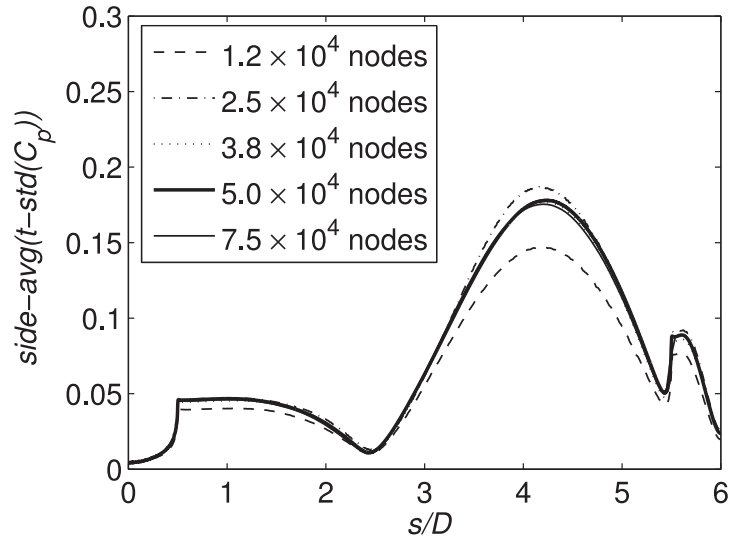

(b)

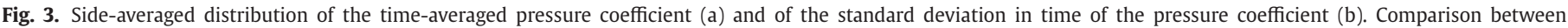
different grid resolutions (RSM).

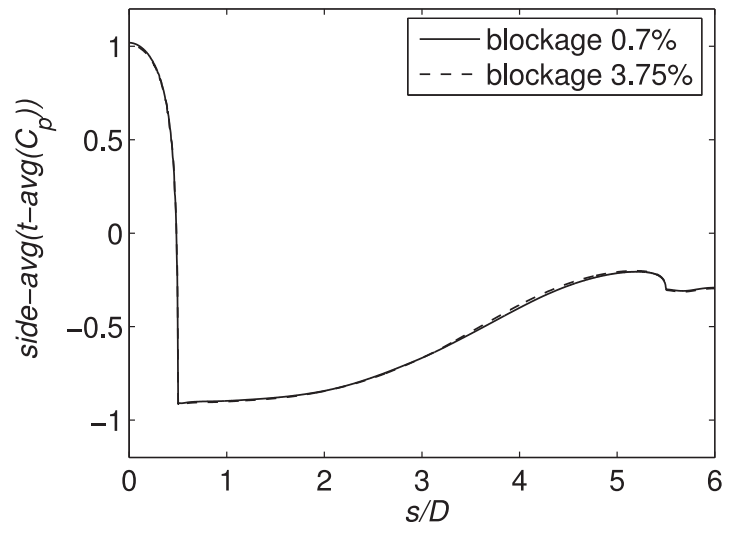

(a)

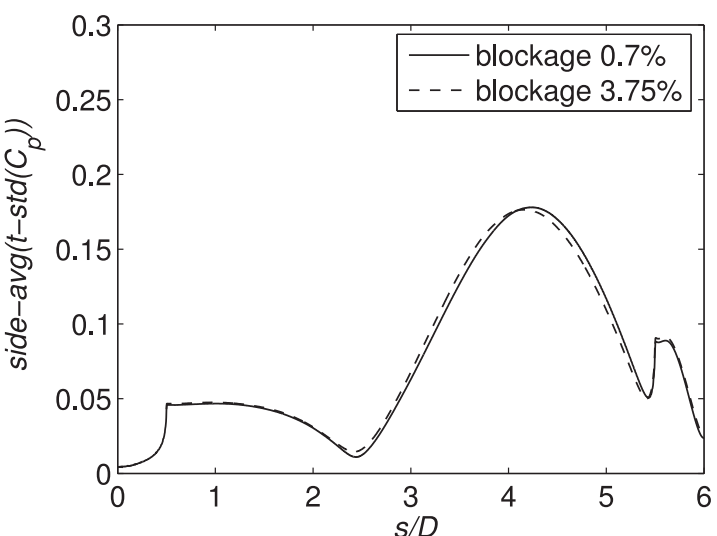

(b)

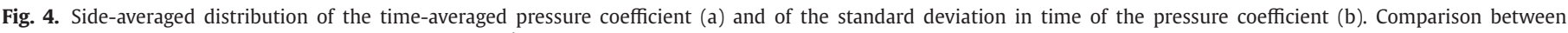
different blockage ratios (RSM and grid having $5.0 \times 10^{4}$ nodes).

discretization errors. In particular, we will also focus on the coarsest grid, i.e. the one having $1.2 \times 10^{4}$ nodes.

\subsection{Sensitivity to blockage}

In the previous simulations, the blockage, i.e. the percentage ratio between $D$ and the computational domain lateral dimension, was $0.7 \%$, which is lower than those of the BARC experimental contributions [5]. To check whether this could have an impact on the results, we carried out an additional simulation with a computational domain having a lateral dimension of $26.66 \mathrm{D}$, corresponding to a blockage of $3.75 \%$, which is the largest value of the BARC experiments. The RSM model and the grid having $5.0 \times$ $10^{4}$ nodes are used. The time-statistic of the aerodynamic loads are compared in Table 3 for the two considered blockage values. Differences lower than $1 \%$ are found for the $t-\operatorname{avg}\left(c_{x}\right)$ and the $t-\operatorname{std}\left(c_{y}\right)$, while the value of $t-\operatorname{avg}\left(c_{y}\right)$ is small in both cases, lower than 0.04 . The side-averaged distributions of the of the timeaveraged $C_{p}$ and of the standard deviation in time of $C_{p}$ are shown in Fig. 4. The blockage has again a negligible effect; the maximum difference is 0.014 for side $-\operatorname{avg}\left(t-\operatorname{avg}\left(C_{p}\right)\right)$ and 0.064 for side $-\operatorname{avg}\left(t-\operatorname{std}\left(C_{p}\right)\right)$.
Table 3

Time statistics of the aerodynamic loads: comparison between different blockage ratios (RSM and grid having $5.0 \times 10^{4}$ nodes).

\begin{tabular}{llll}
\hline Blockage ratios & $t-\operatorname{avg}\left(c_{y}\right)$ & $t-\operatorname{avg}\left(c_{x}\right)$ & $t-\operatorname{std}\left(c_{y}\right)$ \\
\hline $0.7 \%$ & 0.0347 & 1.0921 & 0.6074 \\
$3.75 \%$ & 0.0364 & 1.1026 & 0.6123 \\
\hline
\end{tabular}

\section{Uncertainty quantification methodology}

\subsection{Uncertain parameter selection}

The propagation of the aleatoric uncertainty in three parameters characterizing the incoming flow conditions of the BARC configuration, namely the angle of incidence, the turbulence intensity and length scale, is investigated in the present study.

As previously stated in the Introduction, the choice of these uncertain parameters follows the analysis in [5]. Indeed, difficulties in obtaining a perfect alignment between the model and the incoming flow were reported in two different experimental BARC contributions and they were indicated as a possible source of discrepancies. Furthermore, although the BARC recommends a very low turbulence level in the incoming flow, perfectly smooth flow is 


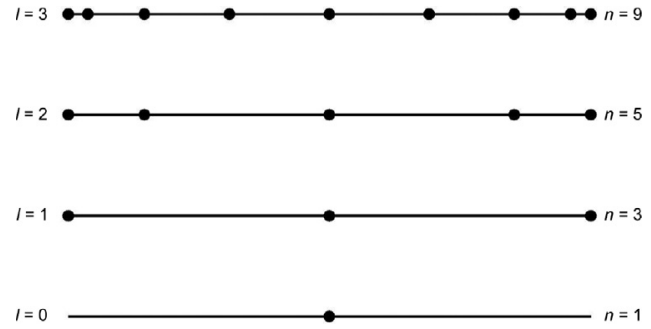

Fig. 5. Nested Clenshaw-Curtis quadrature points at different levels.

impossible to be obtained in experiments at the considered Reynolds number, and a question at issue in the review by [5] was if this could impact the comparison with numerical simulations. To reproduce a turbulent incoming flow in numerical simulations, information on the turbulence intensity, whose value is usually available for wind tunnel experiments, although with some uncertainty, is not sufficient. Additional features of the incoming flow turbulence, such as the turbulence scale, are in most cases not available. Therefore, we consider the inflow turbulence intensity and scale as potentially important sources of uncertainty when trying to compare experiments and simulations. Other uncertainties are present in experiments, which are not considered in the present analysis, e.g. cylinder surface roughness or corner sharpness. Moreover, there are known differences in the set-up of the different experiments, which might also have an impact on some measured quantities, as the model spanwise length. The impact of this parameter is not investigated herein because it would require highly resolved three-dimensional simulations implying huge computational costs. This could be the object of future work.

The following range of variation of the uncertain set-up parameters are investigated: for the angle of incidence $\alpha \in\left[-1^{\circ},+1^{\circ}\right]$, for the longitudinal turbulence intensity $I \in[0.001,0.03]$, and for the turbulence length scale $L \in[0.1 D, 5 D]$. The range of variation of $\alpha$ has been chosen in order to reproduce a small flow misalignment possible in an experiment, while the turbulence intensity variation is slightly larger than the range recommended in the BARC benchmark, but it is well representative of values typically encountered in wind tunnels. Since, as previously said, it is fairly difficult to characterize the turbulence length scale in wind tunnels, especially at low values of $I$, almost no information was available on this quantity from the literature or from the experimental contributions to BARC. Hence, we adopted the quite large range of variation previously specified.

\subsection{Stochastic collocation method}

There are several methodologies to evaluate uncertainties, as shown in [12] and [15]. Most of these methodologies are based on identification, characterization and propagation of input parameter uncertainties. In this study, the Stochastic Collocation (SC) method [19] is employed.

If we consider, first, a single uncertain parameter $\xi$, the SC method is based on interpolating deterministic samples of the quantity of interest, $u(\boldsymbol{x}, t, \xi)$, at quadrature points $\xi_{k}$ using Lagrange polynomials with $L_{j}\left(\xi_{k}\right)=\delta_{j k}$, where $\delta_{j k}$ is the Kronecker delta.

The quadrature points in this study, which correspond to the samples of the uncertain input parameter for which deterministic simulations are carried out, are the Clenshaw-Curtis points. They are nested with $n(l)$ points in level $l: n(l)=2^{l}+1, n(1)=1$ and they are defined as the extrema of the Chebyshev polynomials; $\xi_{k, l}=-\cos (\pi(k-1) /(n(l)-1)), \xi_{k, l}$ being the $k^{\text {th }}$ quadrature point at level $l$ (Fig. 5).
Thus, the interpolation of $u(\boldsymbol{x}, t)$ on quadrature points $\xi$ at level $l$ can be defined as follows:

$P_{l} u(\boldsymbol{x}, t, \xi)=\sum_{j=1}^{n(l)} u\left(\boldsymbol{x}, t, \xi_{j, l}\right) L_{j, l}(\xi), \quad L_{j, l}(\xi)=\prod_{\substack{i=1 \\ i \neq j}}^{n(l)} \frac{\xi-\xi_{i}}{\xi_{j, l}-\xi_{i}}$

By using the interpolation scheme above, different statistical moments can be approximated. For instance, the statistical mean, $\left(\mu_{l} u\right)(\boldsymbol{x}, t)$ can be defined as:

$\left(\mu_{l} u\right)(\boldsymbol{x}, t)=\int_{\Xi} u(\boldsymbol{x}, t, \xi) f(\xi) \mathrm{d} \xi \approx \sum_{k=1}^{n(l)} u\left(\boldsymbol{x}, t, \xi_{k, l}\right) w_{k, l}$

in which $\Xi$ is the parameter space and $f$ is the probability density function of the parameter $\xi$; finally, the quadrature weights $w_{k, l}$ can be expressed as follows:

$w_{k, l}=\int_{\Xi} L_{k, l}(\xi) f(\xi) \mathrm{d} \xi$

Similarly the variance at level $l$ can be computed as:

$\operatorname{Var}(u(\boldsymbol{x}, t)) \approx \sum_{k=1}^{n(l)}\left(u\left(\boldsymbol{x}, t, \xi_{k, l}\right)\right)^{2} w_{k, l}-\left(\sum_{k=1}^{n(l)} u\left(\boldsymbol{x}, t, \xi_{k, l}\right) w_{k, l}\right)^{2}$

The quadrature points are extended to multiple dimensions using Smolyak sparse grids [16]. These reduced grids are based on a weighted linear combination of tensor grids with a relatively small number of quadrature points. In this way, a specific subset of tensor product combinations of 1-D quadrature points is used to construct the sampling points in multi-dimensions. For instance, for $n_{\xi}$ uncertain parameters, the interpolation at level $l$ can be defined as:

$P_{l} u(\boldsymbol{x}, t, \boldsymbol{\xi})=\sum_{\boldsymbol{k} \in L\left(l, n_{\xi}\right)}\left(\Delta P_{k_{1}} \otimes \ldots \otimes \Delta P_{k_{n_{\xi}}}\right) u(\boldsymbol{x}, t, \boldsymbol{\xi})$

where $\xi$ is the vector of the uncertain parameters and the delta formulation in $1 \mathrm{~d}$ is defined as:

$\Delta P_{k} u=\left(P_{k}-P_{k-1}\right) u ; \quad P_{0} u=0$

$P_{k}$ being defined by Eq. (1). The sparse grid subset is defined by:

$\left.L\left(l, n_{\xi}\right)=\left\{\mathbf{k} \in \mathbb{N}_{+}^{n_{\xi}}, \mathbf{k}>\mathbf{1}: l-n_{\xi}+1 \leq \sum_{\delta=1}^{n_{\xi}}\left(k_{\delta}-1\right) \leq l\right)\right\}$

where $\mathbf{k}=\left(k_{1}, k_{2}, \ldots, k_{n_{\xi}}\right)$. For three uncertain parameters, as in the present study, this results in 7 and 25 URANS simulations based on the Smolyak sparse grid extension of the level-1 and level-2 Clenshaw-Curtis quadrature points, respectively (Fig. 6).

The statistical mean based on sparse grid in multi-dimensions can be computed as follows:

$\left(\mu_{l} u\right)(\boldsymbol{x}, t)=\sum_{\boldsymbol{k} \in L\left(l, n_{\xi}\right)}\left(\Delta I_{k_{1}} \otimes \ldots \otimes \Delta I_{k_{n_{\xi}}}\right) u(\boldsymbol{x}, t, \boldsymbol{\xi})$

where $\Delta I_{k}$ is following the delta formulation as in Eq. (6) and is defined as the approximate integral of the interpolated function:

$I_{l} u(\boldsymbol{x}, t, \boldsymbol{\xi})=\sum_{k=1}^{n(l)} w_{k, l} u\left(\boldsymbol{x}, t, \xi_{k, l}\right)$

The sensitivity of the quantities of interest to the single input parameters or to their combinations are quantified using variance decomposition method proposed by [17]; $u(\boldsymbol{x}, t, \boldsymbol{\xi})$ is decomposed in the following form:

$u(\boldsymbol{x}, t, \boldsymbol{\xi})=\sum_{v \subseteq \mathfrak{V}} u_{v}(\boldsymbol{x}, t, \boldsymbol{\xi})$

where $v$ is a subset of $\mathfrak{V}=\left\{1, \ldots, n_{\xi}\right\}$ and $u_{v}(\boldsymbol{x}, t, \boldsymbol{\xi})$ is a component function only dependent on random variables $\xi_{v}=\left\{\xi_{i} \mid \forall i \in v\right\}$. 


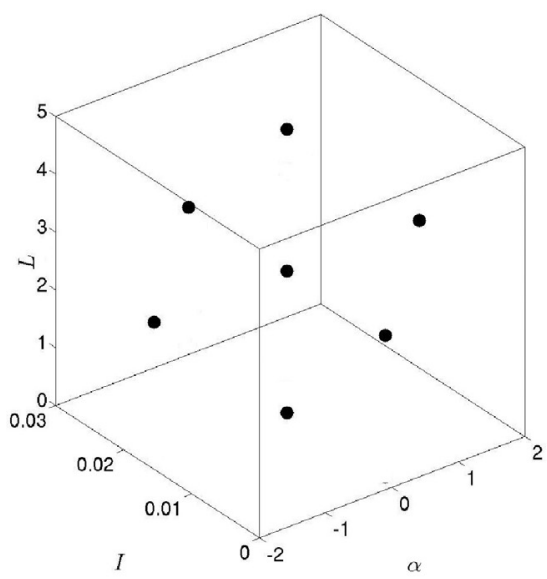

(a)

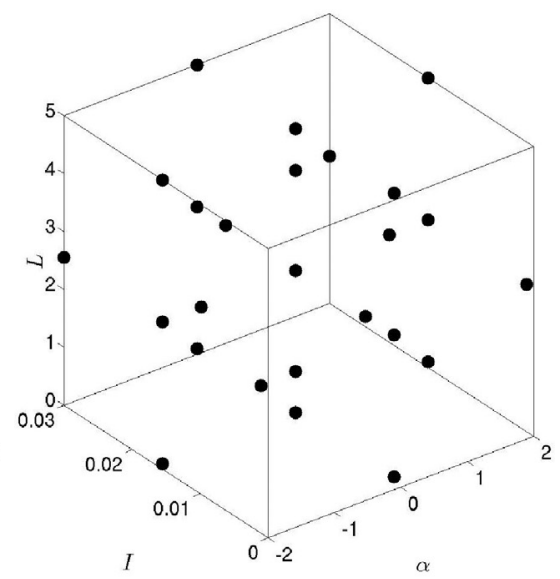

(b)

Fig. 6. Smolyak sparse grid level $=1$ with 7 (a) and level $=2$ with 25 (b) deterministic CFD simulations,

The partial variances $D_{v}$ are defined as following;

$D_{v}=\operatorname{Var}\left(u_{v}\right)=\int u_{v}^{2} d f\left(\xi_{v}\right)$

where $f\left(\xi_{v}\right)$ is the probability density function of $\xi_{v}$.

Considering the orthogonal property of this variance decomposition technique (see [18] and [6]), the partial variance calculation can be rewritten as follows:

$D_{v}=\int\left(\int u d f\left(\xi_{v^{\prime}}\right)\right)^{2} d f\left(\xi_{v}\right)-\sum_{r \subset v} D_{r}$

in which $v^{\prime}$ is the complement of $v$, defined such that $\left\{v \cup v^{\prime}\right\}=\mathfrak{V}$ and $\left\{v \cap v^{\prime}\right\}=\varnothing$ and $r$ is a subset of $v$. Consequently, the sensitivity index can be defined as the ratio of partial variances to the total variance, $D_{\text {tot }}=\operatorname{Var}(u)=\sum_{v \subseteq \mathfrak{V}} D_{v}$ :

$S_{v}=\frac{D_{v}}{D_{t o t}}$

Tang et al. [18] described the calculation of Sobol indices on tensor grids. The integrals in Eq. (12) need to be approximated for calculation of Sobol indices. The first term in Eq. (12) is approximated by splitting the contribution of $v$ and $v^{\prime}$ subset as follows (more information and derivations can be found in 6):

$$
\begin{aligned}
& \int u d f\left(\xi_{v^{\prime}}\right) \approx \int P_{l} u d f\left(\xi_{v^{\prime}}\right) \\
& \quad \approx \sum_{\substack{\boldsymbol{k}_{v} \\
\boldsymbol{k}_{v}^{\prime}}}\left(\Delta P_{k_{v_{1}}} \otimes \ldots \otimes \Delta P_{k_{v_{p}}}\right) u \otimes\left(\Delta I_{k_{v_{1}^{\prime}}} \otimes \ldots \otimes \Delta I_{k_{v_{m}^{\prime}}}\right) \\
& u=P_{l} u_{v} \otimes I_{l} u_{v^{\prime}}
\end{aligned}
$$

Finally, using Eqs. (12) and (14), the partial variances can be computed as follows (see 18):

$D_{v} \approx\left(I_{l} u_{v^{\prime}}\right)^{2} \otimes I_{l} u_{v}^{2}-\sum_{r \subset v} D_{r}$

The effect of the above-mentioned uncertainties on the quantities of interest is quantified by estimating stochastic means, standard deviations and partial variances of the time statistics of the aerodynamics loads, of the surface pressure coefficient distribution, and of the velocity and pressure fields.

\section{Definition of the text matrix and convergence of the UQ procedure}

Initially, uniform input probability distributions are used. The choice of this input probability distribution is justified by the least informative distribution with the highest variance in the intervals obtained from available data and expert judgements. The previously described UQ procedure, based on the Smolyak sparse grid extension of the level-2 Clenshaw-Curtis quadrature points, results in 25 URANS simulations. The UQ procedure convergence is estimated by comparing the results with those on the nested lower levels (Fig. 6). The values of the set-up parameters for these 25 deterministic simulations are reported in Table 4, together with the obtained time-statistics of the aerodynamic loads, namely $t-$ $\operatorname{avg}\left(c_{y}\right), t-\operatorname{avg}\left(c_{x}\right)$ and $t-\operatorname{std}\left(c_{y}\right)$.

The stochastic mean and standard deviation of the aerodynamic load time-statistics are given in Table 5 for different levels of the stochastic collocation procedure. The RSM is considered on the grid having $5.0 \times 10^{4}$ nodes, for which grid independence is reached (see Section 3.4). The Cumulative Distribution Functions (CDF) of the previous quantities are given in Fig. 7 for the same levels. The differences between stochastic collocation results obtained at level 1 and these at level 2 are in general acceptable. In particular, the stochastic mean values of $t-\operatorname{avg}\left(c_{x}\right)$ and $t-\operatorname{std}\left(c_{y}\right)$ are very well converged, the differences being lower than $1 \%$. As for the stochastic mean of $t-\operatorname{avg}\left(c_{y}\right)$, there is no clear converge with increasing the level. We accept this behavior, since the values the stochastic mean of this quantity, which should be zero, remain low (see also the discussion in Sections. 3.2 and 3.4). The differences in the stochastic standard deviations between levels 1 and 2 are larger for $t-\operatorname{avg}\left(c_{x}\right)$ and $t-\operatorname{std}\left(c_{y}\right)$, up to $11 \%$ for $t-\operatorname{std}\left(c_{y}\right)$. The maximum percent deviation in the CDF of this quantity is indeed $12.7 \%$; note that it is localized in the largest values of the CDF, corresponding to the tails of the PDF. Conversely the stochastic standard deviation of $t-\operatorname{avg}\left(c_{y}\right)$ seems to be well converged (the difference is $2 \%$ ).

Finally, Fig. 8 shows the side-averaged distribution of the time average and of the standard deviation in time of the pressure coefficient; in particular, the thick lines are the stochastic mean of these quantities, while the shaded areas, delimited by thin lines, represent the stochastic mean \pm the stochastic standard deviation. The differences between level 1 and 2 of the stochastic collocation procedure are small for all the quantities shown in Fig. 8. More quantitatively, the maximum deviations are 0.0036 and 0.0025 for the stochastic means of the time-average and time standard deviation of $C_{p}$ respectively and 0.0062 and 0.0043 for the stochastic standard deviation of the same quantities. Based on the previous analysis, also considering the significant costs of a third level, all the UQ results shown in the 


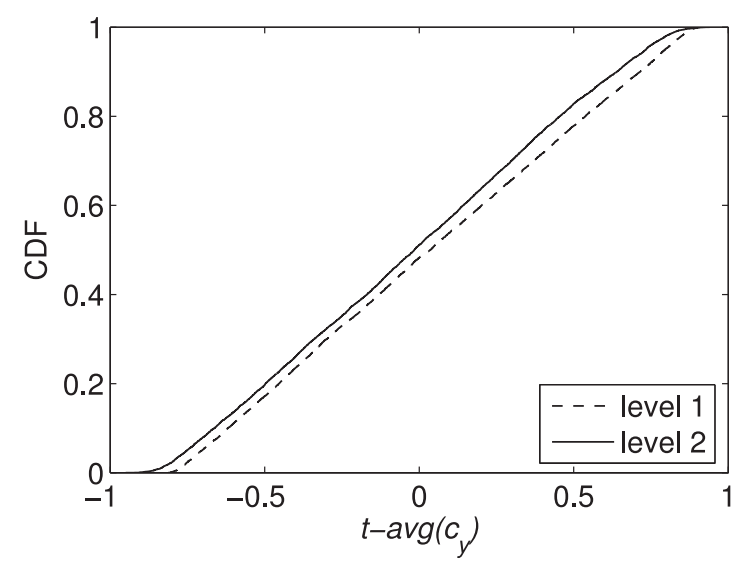

(a)

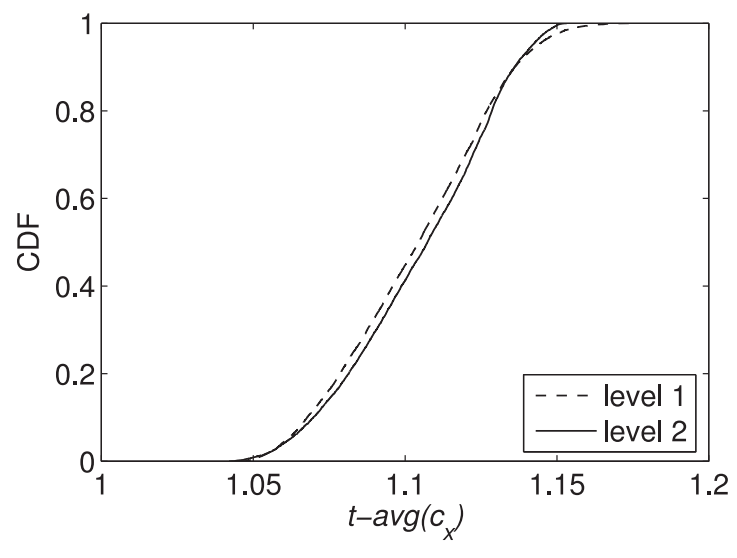

(b)

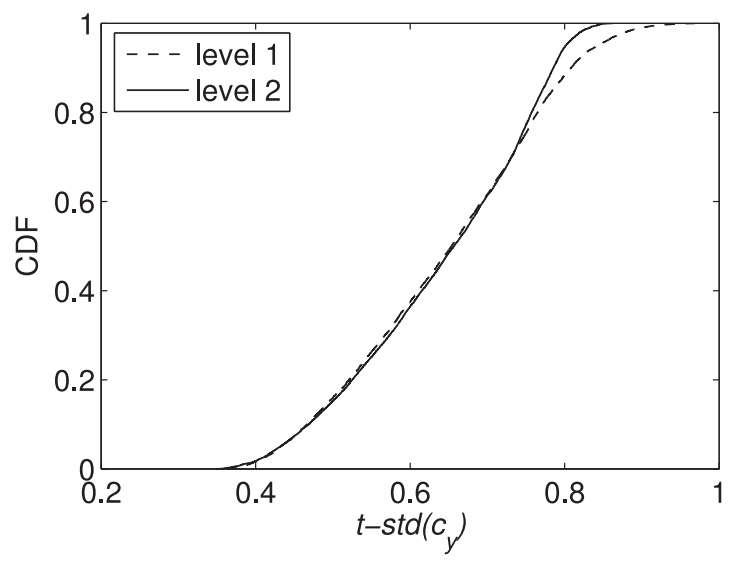

(c)

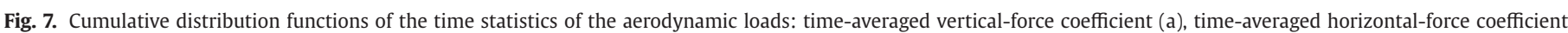

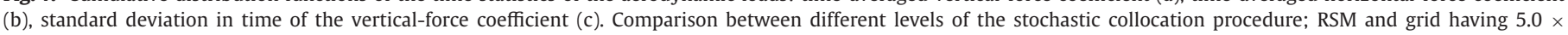
$10^{4}$ nodes.

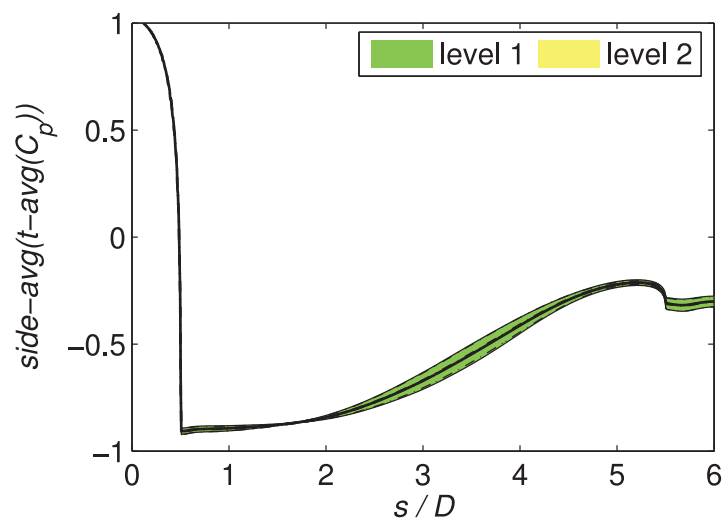

(a)

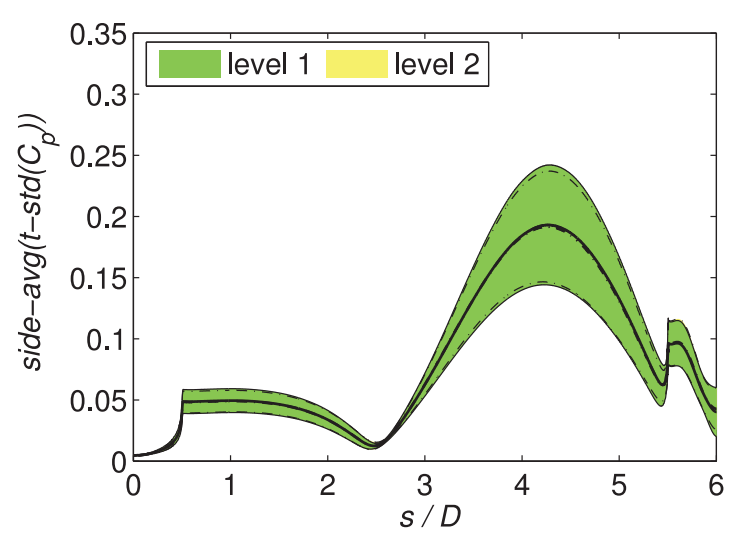

(b)

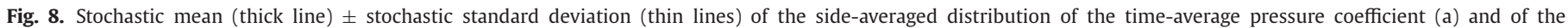

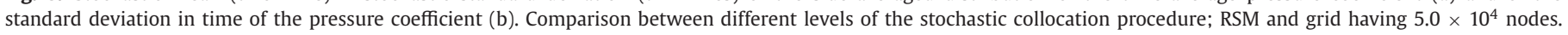


Table 4

Level-2 Clenshaw-Curtis quadrature points in the parameter space and time statistics of the aerodynamic loads obtained in the corresponding deterministic URANS simulations; RSM and grid having $5.0 \times 10^{4}$ nodes.

\begin{tabular}{llllrll}
\hline Case & $\alpha[\circ]$ & $I[\%]$ & $L[D]$ & $t-\operatorname{avg}\left(c_{y}\right)$ & $t-\operatorname{avg}\left(c_{x}\right)$ & $t-\operatorname{std}\left(c_{y}\right)$ \\
\hline 1 & 0 & 0.0155 & 2.5500 & 0.0347 & 1.0921 & 0.6074 \\
2 & -1.0000 & 0.0155 & 2.5500 & -0.7940 & 1.1118 & 0.6758 \\
3 & 1.0000 & 0.0155 & 2.5500 & 0.8818 & 1.1192 & 0.6971 \\
4 & -0.7071 & 0.0155 & 2.5500 & -0.5502 & 1.1037 & 0.6395 \\
5 & 0.7071 & 0.0155 & 2.5500 & 0.5989 & 1.1115 & 0.6574 \\
6 & 0 & 0.0010 & 2.5500 & 0.0680 & 1.1272 & 0.7789 \\
7 & 0 & 0.0300 & 2.5500 & 0.0173 & 1.0482 & 0.3756 \\
8 & -1.0000 & 0.0010 & 2.5500 & -0.7989 & 1.1504 & 0.8192 \\
9 & 1.0000 & 0.0010 & 2.5500 & 0.9640 & 1.1505 & 0.8406 \\
10 & -1.0000 & 0.0300 & 2.5500 & -0.7358 & 1.0622 & 0.4469 \\
11 & 1.0000 & 0.0300 & 2.5500 & 0.7881 & 1.0634 & 0.4520 \\
12 & 0 & 0.0052 & 2.5500 & 0.0550 & 1.1239 & 0.7628 \\
13 & 0 & 0.0258 & 2.5500 & 0.0203 & 1.0650 & 0.4521 \\
14 & 0 & 0.0155 & 0.1000 & 0.0421 & 1.1197 & 0.7419 \\
15 & 0 & 0.0155 & 5.0000 & 0.0281 & 1.0947 & 0.6089 \\
16 & -1.0000 & 0.0155 & 0.1000 & -0.8127 & 1.1419 & 0.7906 \\
17 & 1.0000 & 0.0155 & 0.1000 & 0.9234 & 1.1449 & 0.8025 \\
18 & -1.0000 & 0.0155 & 5.0000 & -0.8059 & 1.1141 & 0.6827 \\
19 & 1.0000 & 0.0155 & 5.0000 & 0.8769 & 1.1179 & 0.6941 \\
20 & 0 & 0.0010 & 0.1000 & -0.0601 & 1.1270 & 0.7790 \\
21 & 0 & 0.0300 & 0.1000 & -0.0413 & 1.1140 & 0.7051 \\
22 & 0 & 0.0010 & 5.0000 & -0.0683 & 1.1272 & 0.7789 \\
23 & 0 & 0.0300 & 5.0000 & -0.0245 & 1.0463 & 0.3667 \\
24 & 0 & 0.0155 & 0.8176 & -0.0392 & 1.1000 & 0.6398 \\
25 & 0 & 0.0155 & 4.2824 & -0.0325 & 1.0913 & 0.6025 \\
\hline & & & & & &
\end{tabular}

Table 5

Stochastic mean and standard deviation of the time statistics of the aerodynamic loads for different levels of the stochastic collocation procedure; RSM and grid having $5.0 \times$ $10^{4}$ nodes.

\begin{tabular}{lcll}
\hline & $t-\operatorname{avg}\left(c_{y}\right)$ & $t-\operatorname{avg}\left(c_{x}\right)$ & $t-\operatorname{std}\left(c_{y}\right)$ \\
\hline Stochastic mean (level 0) & 0.0347 & 1.0921 & 0.6074 \\
Stochastic mean (level 1) & 0.0405 & 1.1035 & 0.6464 \\
Stochastic mean (level 2) & -0.0155 & 1.1053 & 0.6426 \\
Stochastic standard deviation (level 0) & 0 & 0 & 0 \\
Stochastic standard deviation (level 1) & 0.4840 & 0.0267 & 0.1322 \\
Stochastic standard deviation (level 2) & 0.4740 & 0.0246 & 0.1183 \\
\hline
\end{tabular}

following sections are at level 2 of the stochastic collocation procedure.

\section{UQ results for the Reynolds stress model at grid independence}

\subsection{Time statistics of aerodynamic loads and pressure field}

Fig. 9 shows the stochastic mean \pm the stochastic standard deviation of the following quantities: time-averaged horizontalforce coefficient, time-averaged vertical-force coefficient and standard deviation in time of the vertical-force coefficient. The stochastic mean value of the time-averaged horizontal-force coefficient (1.105) is in good agreement with the ensemble average of the contributions to BARC (1.074) and with the available experimental data (1.103), while the stochastic variation is very small, narrower than the already limited dispersion observed among the numerical contributions to BARC. Indeed, the stochastic standard deviation of $t-\operatorname{avg}\left(c_{x}\right)$ is 0.024 , while the BARC ensemble standard deviation is 0.129 (5). The stochastic mean of the time-averaged vertical-force coefficient is close to zero, as expected; its stochastic variation is quite large, much larger than the variation of this quantity previously observed with varying statistic sample, grid resolution, stochastic level or blockage. The stochastic variation of $t-\operatorname{avg}\left(c_{y}\right)$ is mainly due to the fact that the angle of attack is allowed to assume values up to $\pm 1^{\circ}$ (see also Section 6.3). Conversely, the stochastic variation of $t-\operatorname{std}\left(c_{y}\right)(0.118)$ is significantly lower than the standard deviation computed in [5] over the different numerical contributions to BARC (0.374). These results are a first indication that the impact of the uncertainties in the considered set-up parameters is smaller than that of other sources of errors/uncertainties, namely turbulence modeling, numerics, computational set-up.

The distribution along the cylinder side of the side-averaged and time-averaged pressure coefficient is given in Fig. 10. As previously, the stochastic mean value \pm the stochastic standard deviation are shown; they are compared with the ensemble statistics of the different experimental and CFD contributions to BARC (data from [5]). The stochastic variation of side $-\operatorname{avg}\left(t-\operatorname{avg}\left(C_{p}\right)\right)$ is significantly lower than the dispersion of the BARC simulations and, even more, of the experimental studies. To provide a more quantitative comparison, we computed the ensemble standard deviations of the experimental and numerical BARC data along the cylinder surface; they are shown and compared with the stochastic standard deviation in Fig. 10(c). It can be seen that the stochas- 


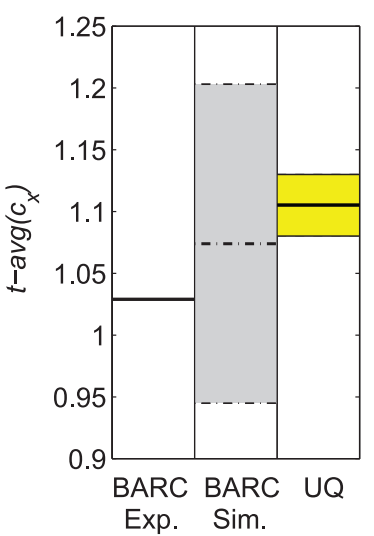

(a)

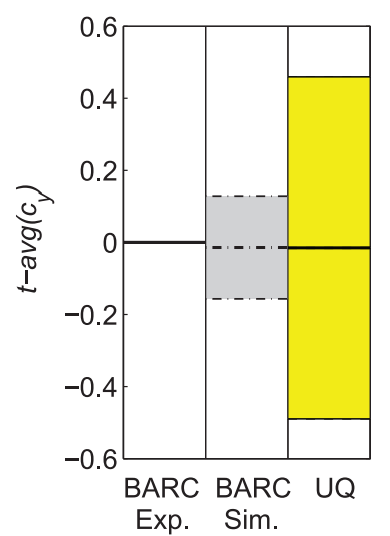

(b)

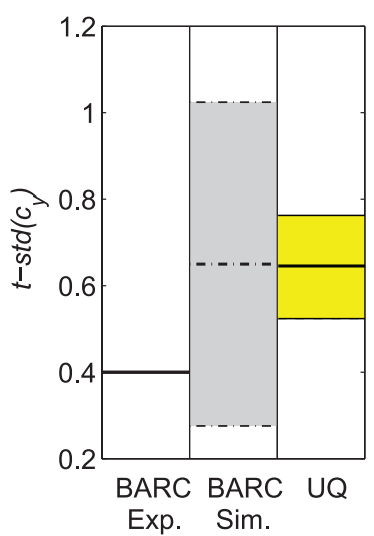

(c)

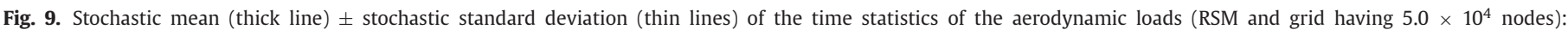

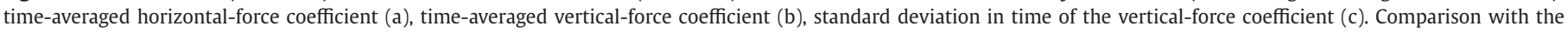
ensemble average and standard deviation of the numerical contributions to BARC [5] and with the experiments by [13,14].

tic standard deviation is always lower than the ensemble standard deviations of BARC contributions. In particular, the maximum value of the stochastic standard deviation is 0.041, while those of the ensemble standard deviation of BARC experiments and simulations are 0.214 and 0.165 respectively. This confirms the previous observations made for the time statistics of the aerodynamic loads, indicating that the considered uncertainties in the set-up parameters, which may be present in experiments, can not solely explain the dispersion of the BARC experimental data.

Fig. 11 shows the same comparisons as in Fig. 10 for the sideaveraged standard deviation in time of $C_{p}$, side $-\operatorname{avg}\left(t-\operatorname{std}\left(C_{p}\right)\right)$. The stochastic variation is more significant for this quantity, especially in the peak zone near the flow reattachment point. This agrees with the previous BARC findings which indicate that this is one of the most dispersed quantities of interest. However, the size of the stochastic uncertainty range is again smaller than the dispersion observed among the different BARC data. Note that, the stochastic mean distribution has a different shape than that of the ensemble average of the experimental and CFD contributions to BARC; the main difference is that the ensemble average of the contributions to BARC has a single peak along the cylinder side, while in the stochastic mean a minimum is also found at a distance of approximately $2 D$ from the upstream corner. This is however a behavior typical of URANS simulations, as observed in [5] and shown in Fig. 11(c). A more quantitative comparison is provided in Fig. 11(d), showing the stochastic standard deviation of side $-\operatorname{avg}\left(t-\operatorname{std}\left(C_{p}\right)\right)$ vs. the ensemble standard deviations of the same quantity for the experimental, numerical and URANS contributions to BARC. Once again the stochastic standard deviation is lower than those of the BARC contributions on the whole cylinder lateral surface; the maximum values are 0.046 for the stochastic standard deviation, $0.068,0.108$ and 0.124 for the experimental, numerical and URANS contributions to BARC respectively.

The stochastic mean and standard deviation of the time-average and of the time standard deviation of $C_{p}$ in the whole flow field are shown in Fig. 12. The uncertainty in the time-averaged pressure coefficient has its maximum on the cylinder side in the zone where it has a significant gradient, which corresponds to the change of curvature of the mean streamlines in the rear part of the main flow separation zone on the cylinder side (see Fig. 2). These results agree with those relative to side $-\operatorname{avg}\left(t-\operatorname{avg}\left(C_{p}\right)\right)$ on the cylinder side of Fig. 10 previously commented. The stochastic standard deviation of side $-\operatorname{avg}\left(t-\operatorname{std}\left(C_{p}\right)\right)$ is again significant on the cylinder side, in the zone in which side $-\operatorname{avg}\left(t-\operatorname{std}\left(C_{p}\right)\right)$ has a peak, which is also the one in which the largest dispersion has been observed in the BARC contributions (5). However, the largest stochastic uncertainty in the time standard deviation of pressure coefficient is in the near wake where there is vortex shedding and this is probably due to changes in the wake topology occurring when the angle of attack is changed.

\subsection{Time statistics of the velocity field}

Fig. 13 shows the stochastic mean and standard deviation of the time average and time standard deviation of the velocity magnitude. It can be seen that the largest uncertainty in the mean velocity magnitude is in the shear-layers detaching from the upstream corners and in the zone in which the mean flow reattaches on the cylinder side. This means that the size of the detached zone on the cylinder side is significantly sensitive to the considered uncertain parameters. The BARC predictions of the location of flow reattachment and of the size and shape of the mean recirculation zone on the cylinder side were also found to be largely dispersed (5). In order to better quantify this variability, the reattachment point location was identified in each simulation and uncertainty quantification was carried out also for this quantity. To provide a consistent comparison with the results shown in [5], we carried out the UQ analysis for the average of the coordinate $x / D$ of the reattachment point on the top $(y / D=0.5)$ and bottom $(y / D=-0.5)$ cylinder surfaces, although this smooths the asymmetries in the cases in which the incidence is not equal to zero. The results are shown in Fig. 14, showing the stochastic mean \pm standard deviation of the reattachment point $x / D$ coordinate, compared with the ensemble average and standard deviations of the numerical contributions reviewed in [5]. The available experimental data in [5] is also reported. The stochastic mean values obtained in the present simulations are noticeably lower than the experimental value and of the ensemble average of the BARC contributions, being even outside of the variability range of the BARC results. This is probably due to the RSM turbulence model, as it will be shown in Section 8 . The stochastic variance is once again lower than the global dispersion of the BARC results, confirming that, also for the location 


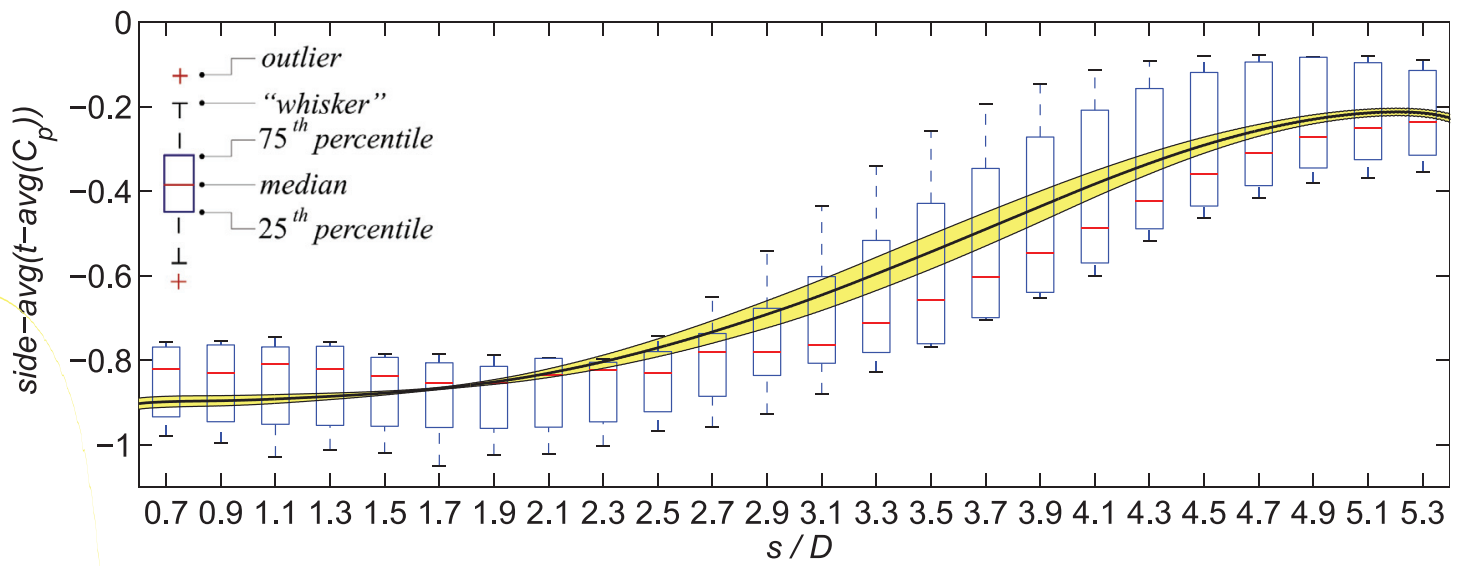

(a)

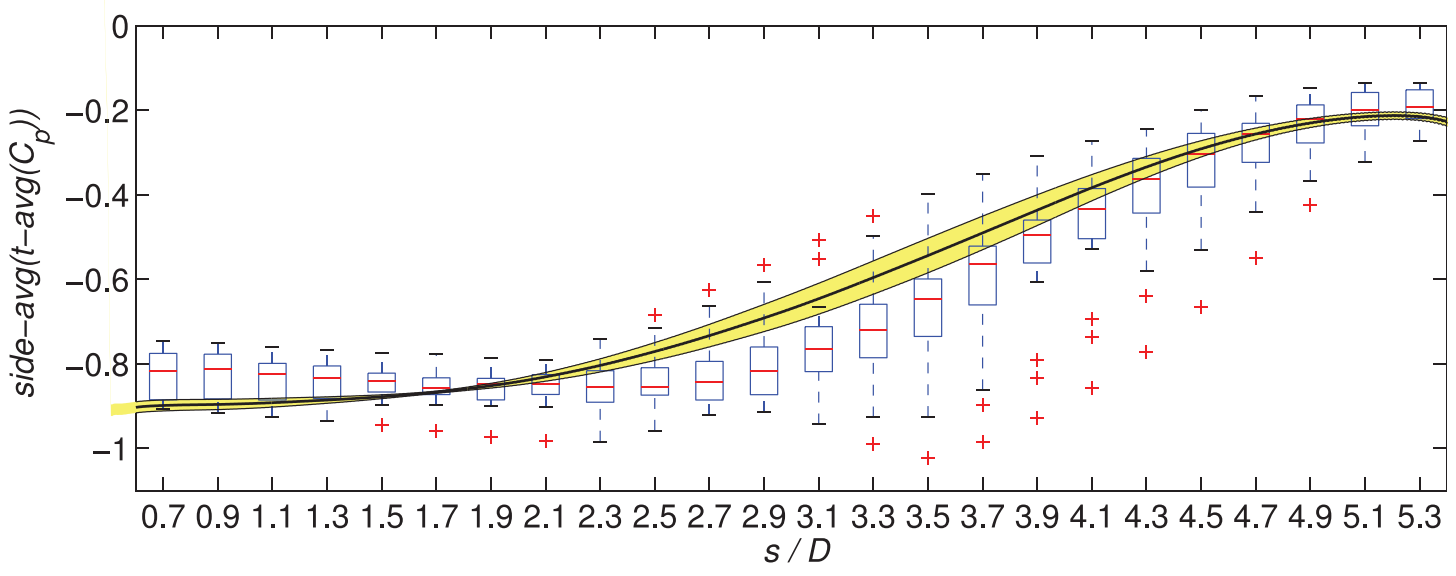

(b)

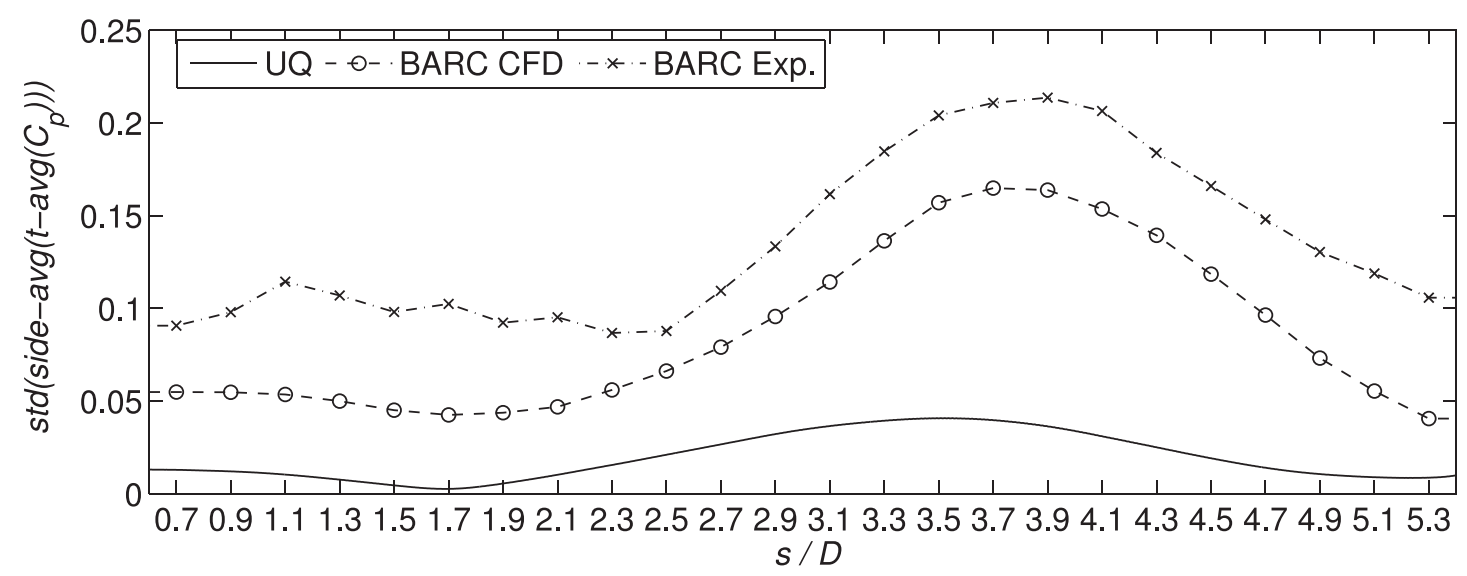

(c)

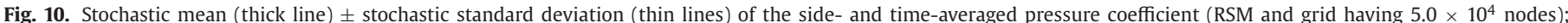

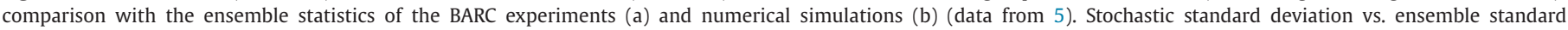
deviation of BARC contributions (c). 


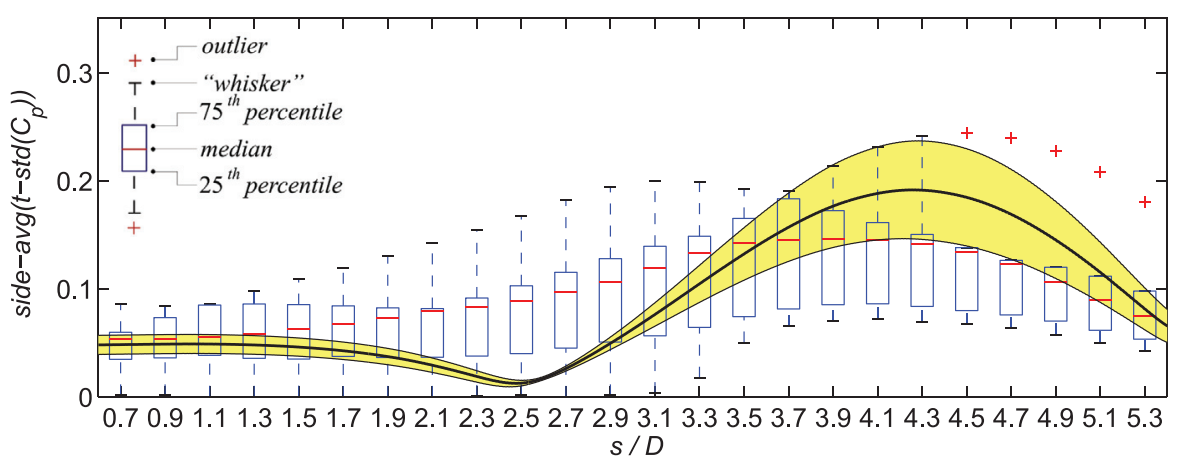

(a)

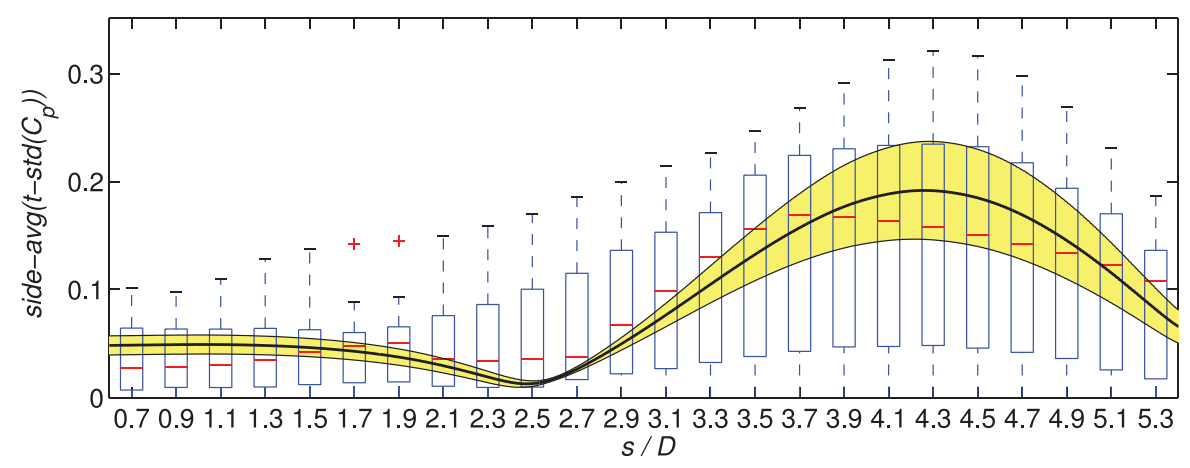

(b)

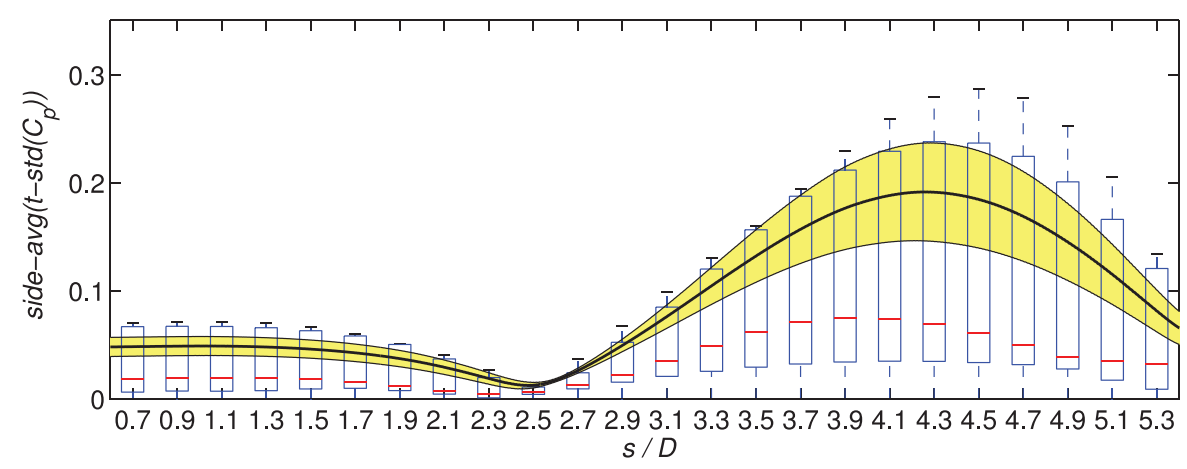

(c)

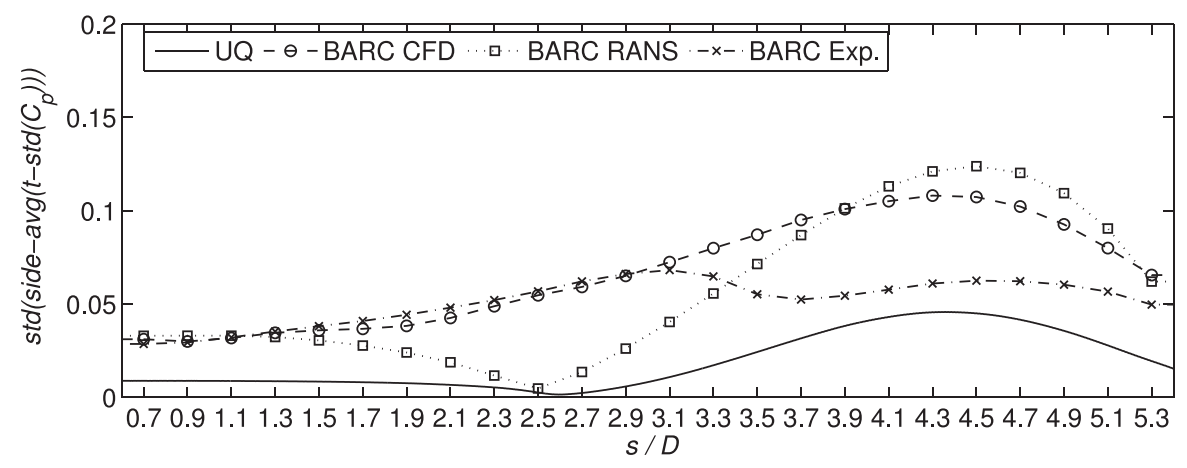

(d)

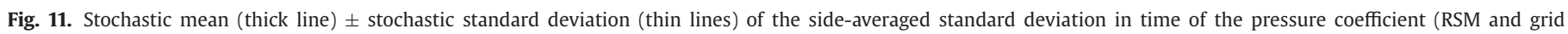

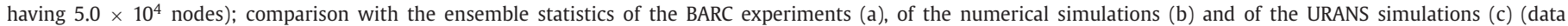
from 5). Stochastic standard deviation vs. ensemble standard deviation of BARC contributions (d). 


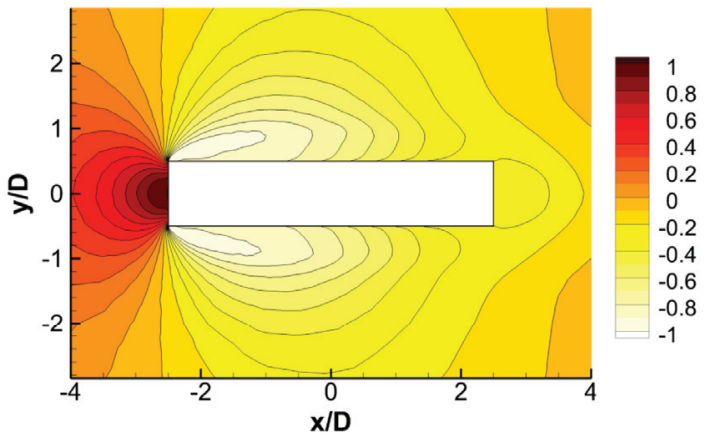

(a) Stochastic mean of the time-averaged pressure coefficient

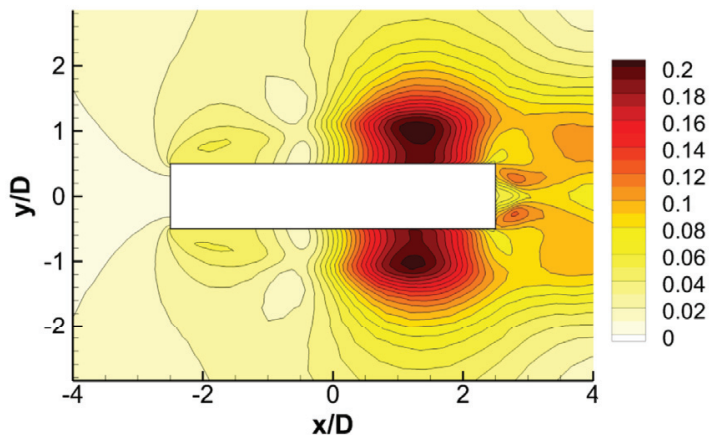

(c) Stochastic mean of the time-standarddeviation pressure coefficient

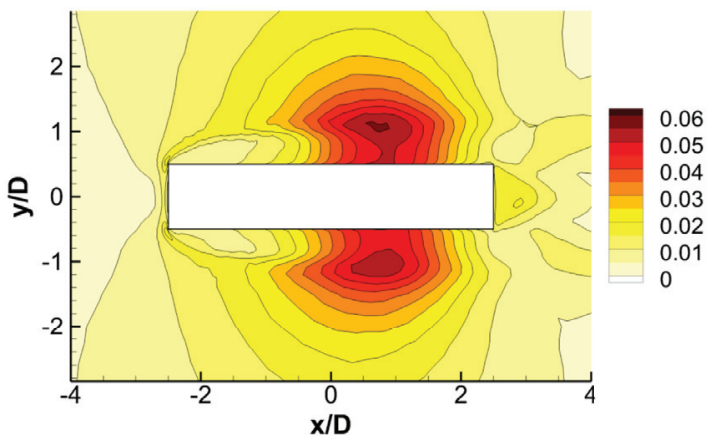

(b) Stochastic standard deviation of the time-averaged pressure coefficient

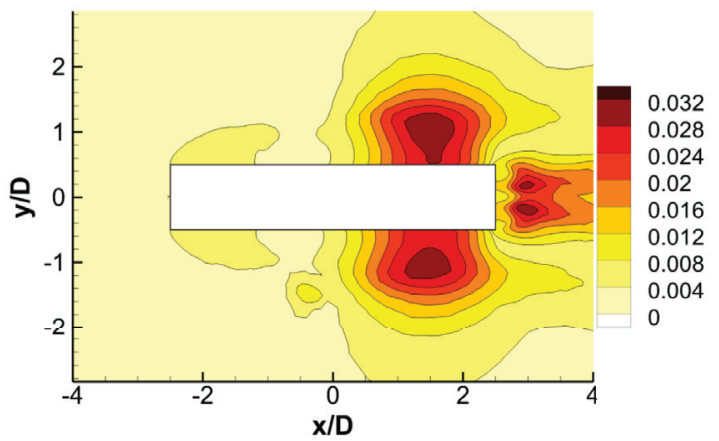

(d) Stochastic standard deviation of the time-standard-deviation pressure coefficient

Fig. 12. Stochastic mean and standard deviation of the time-average and of the time-standard-deviation of pressure coefficient. RSM and grid having $5.0 \times 10^{4}$ nodes.

Table 6

Stochastic variance decomposition of the time statistics of the aerodynamic loads; RSM and grid having $5.0 \times 10^{4}$ nodes.

\begin{tabular}{llllllll}
\hline & $S_{\alpha}$ & $S_{I}$ & $S_{L}$ & $S_{\alpha-I}$ & $S_{\alpha-L}$ & $S_{I-L}$ & $S_{\alpha-I-L}$ \\
\hline$t-\operatorname{avg}\left(c_{x}\right)$ & 0.090 & 0.762 & 0.079 & 0.002 & 0 & 0.067 & 0 \\
$t-\operatorname{avg}\left(c_{y}\right)$ & 0.994 & 0 & 0.004 & 0.002 & 0 & 0.001 & 0 \\
$t-\operatorname{std}\left(c_{y}\right)$ & 0.034 & 0.829 & 0.065 & 0.001 & 0.001 & 0.070 & 0 \\
\hline
\end{tabular}

of the reattachment point, the considered uncertainties do not explain the observed dispersion of the BARC results.

\subsection{Variance decomposition}

The partial variances of quantities of interest to the different uncertain parameters are analyzed in this section. As previously, the simulations with RSM on the grid having $5 \times 10^{4}$ nodes and level 2 for the UQ procedure are considered. The partial variances for the time statistics of the aerodynamic loads are reported in Table 6 as a fraction of the total stochastic variance. The partial variances are the stochastic variances due to the uncertainties in the single set-up parameters, $\alpha, I, L$, and to their interaction, $\alpha-I$, $\alpha-L, I-L, \alpha-I-L$. As expected, the angle of incidence $\alpha$ is the only parameter significantly influencing the $t-\operatorname{avg}\left(c_{y}\right)$. Conversely, the freestream turbulence intensity $I$ is the most impor- tant parameter for the $t-\operatorname{avg}\left(c_{x}\right)$ and the $t-\operatorname{std}\left(c_{y}\right)$. Therefore, this parameter should be carefully reproduced in numerical simulations to have consistent comparison with the experiments. The freestream turbulence length scale $L$, which is difficult to be characterized in experiments, is the least important parameter. Indeed, it has a significant impact only on the variance of $t-\operatorname{avg}\left(c_{x}\right)$, which, however, is characterized by a very low total stochastic variance. As for the combined impact of the uncertainties in the different parameters, only $I$ and $L$ have a significant interaction, as could have been expected, since they both characterize the freestream turbulence.

The partial stochastic variances of the time average and standard deviation of the surface pressure coefficient, due to the single set-up parameters, are shown in Fig. 15. The angle of attack $\alpha$ is the most important parameter for the side $-\operatorname{avg}\left(t-\operatorname{avg}\left(C_{p}\right)\right)$. On the cylinder base the sensitivity to $\alpha$ decreases, indicating that the vortex shedding behind the cylinder is not considerably affected by the angle of attack. Conversely, it is known that the turbulence intensity influences the characteristics of the vortex shedding and of the near wake and this is consistent with the augmented stochastic sensitivity to $I$. On the other hand, the inlet turbulence intensity $I$ is the most dominant parameter on the variation of side - avg $(t-$ $\left.\operatorname{std}\left(C_{p}\right)\right)$. Only near the local minimum of side $-\operatorname{avg}\left(t-\operatorname{std}\left(C_{p}\right)\right)$, around $s / D=2.5$ (see Fig. 11), the sensitivity to the turbulence intensity significantly drops, $\alpha$ becoming the most important param- 

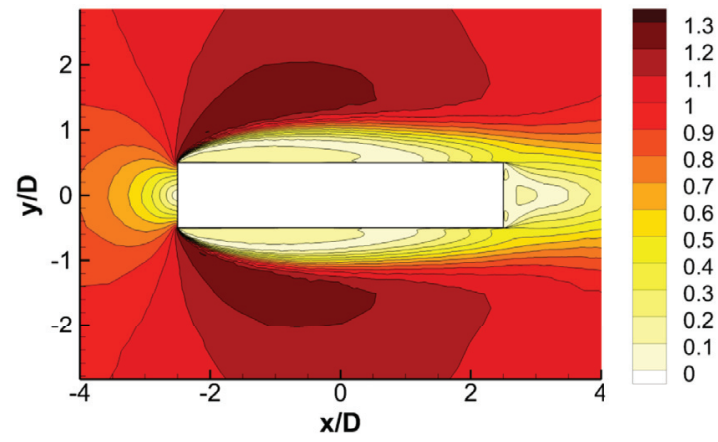

(a) Stochastic mean of the time-averaged velocity magnitude

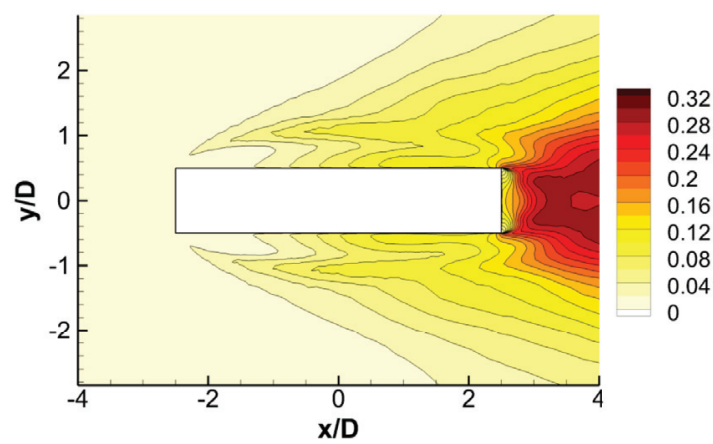

(c) Stochastic mean of the time-standarddeviation velocity magnitude

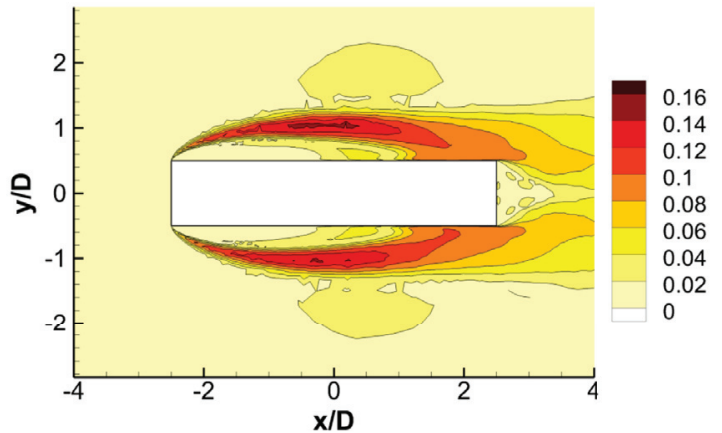

(b) Stochastic standard deviation of the time-averaged velocity magnitude

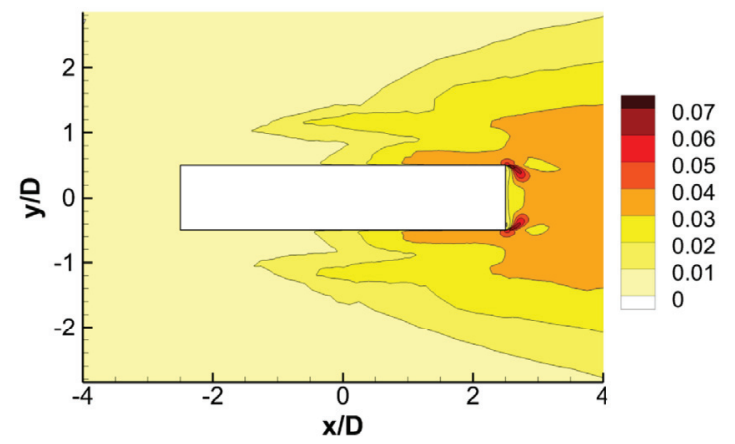

(d) Stochastic standard deviation of the time-standard-deviation velocity magnitude

Fig. 13. Stochastic mean and standard deviation of the time-average and time-standard-deviation of velocity magnitude. RSM and grid having $5.0 \times 10^{4}$ nodes.

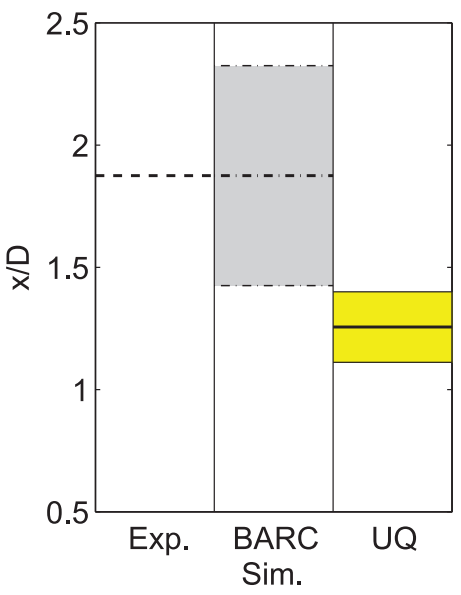

Fig. 14. Stochastic mean (thick line) \pm stochastic standard deviation (thin lines) of the side-averaged streamwise coordinate of the mean flow reattachment point (RSM and grid having $5.0 \times 10^{4}$ nodes); comparison with the ensemble statistics of the BARC results and with the experimental data (data from 5 ).

eter. However, it can be seen in Fig. 11 that in this zone the global stochastic variance of side $-\operatorname{avg}\left(t-\operatorname{std}\left(C_{p}\right)\right)$ is very low, confirming that the sensitivity of this quantity to $\alpha$ is actually low everywhere.

\section{Analysis of the input PDF effect on the UQ results}

The choice of uniform distribution for set-up parameters was justified by lack of information on distribution functions. However, in this Section UQ results are shown for two additional different input probability distributions, to investigate the impact of the choice of the set-up parameter PDF on the aleatoric uncertainty propagation. Note that the PDF of the input parameters must be chosen a-priori and, in this case, no information is available on their shape. A beta distribution with shape parameters $\alpha=\beta=4$ is chosen because it resembles a normal distribution, bounded in the same interval as the original uniform distribution. A uniform distribution with the reduced variance equal to variance of the chosen beta distribution is also considered, in order to isolate the distribution shape effect. The standard deviation of the considered beta distribution is $1 / 3 \approx 0.333$, while the standard deviation of the original uniform distribution on the normalized interval $[-1,1]$ is $1 / \sqrt{3} \approx 0.577$. Fig. 16 (a) shows the different types of input probability distributions rescaled on the interval $[-1,1]$; those actually used for the input parameters can be easily obtained by proper rescaling to the considered ranges of variation and relevant normalization. The related cumulative distributions are also shown in Fig. 16(b).

The effect of different distribution functions on the time statistics of the aerodynamic loads is shown in Fig. 17. As can be seen, there is practically no difference between the results for the beta 


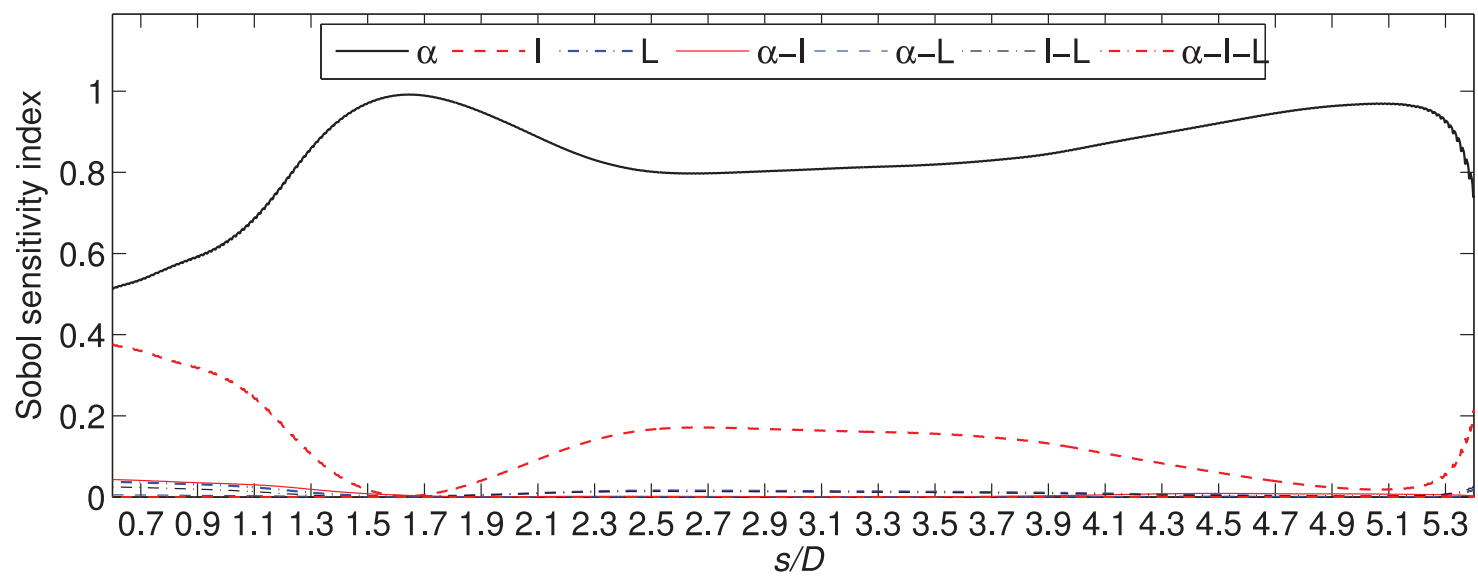

(a)

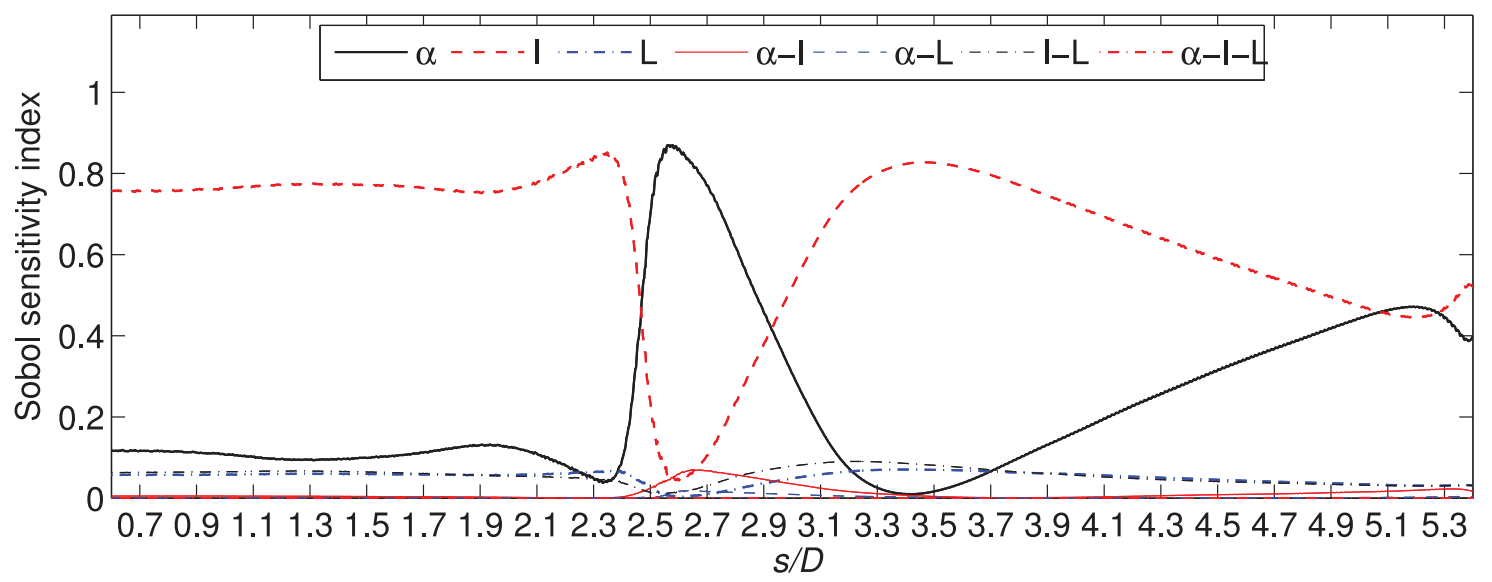

(b)

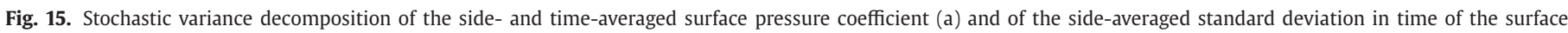
pressure coefficient (b) (RSM and grid having $5.0 \times 10^{4}$ nodes).

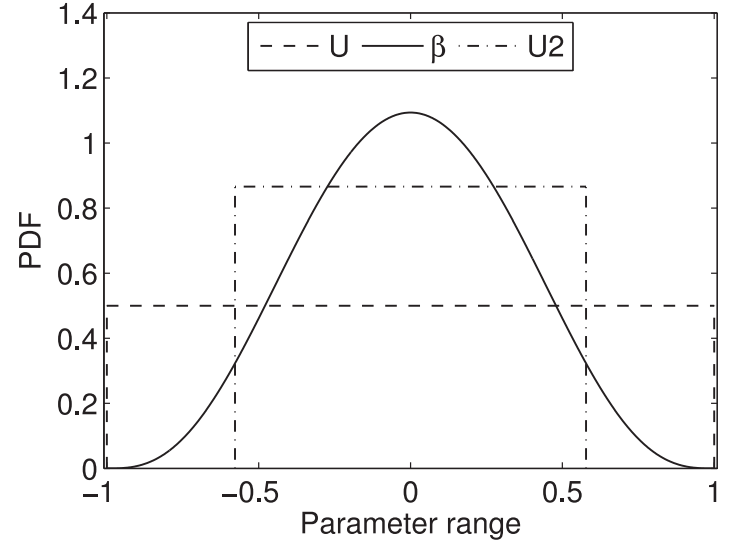

(a) PDF

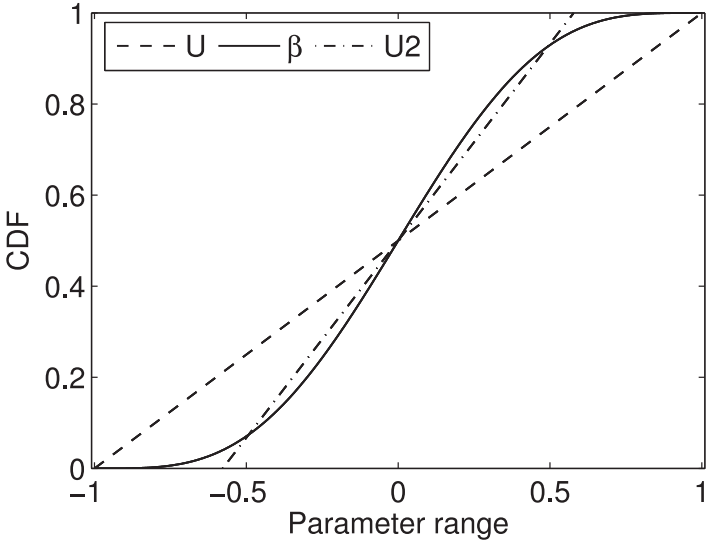

(b) $\mathrm{CDF}$

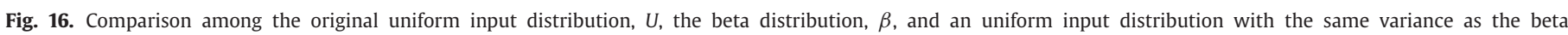
distribution, $U 2$. 


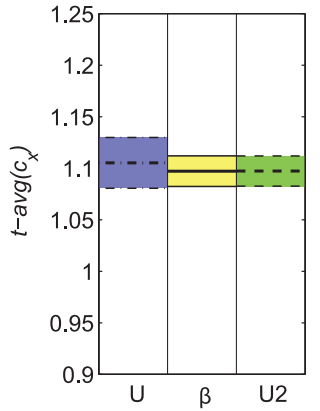

(a)

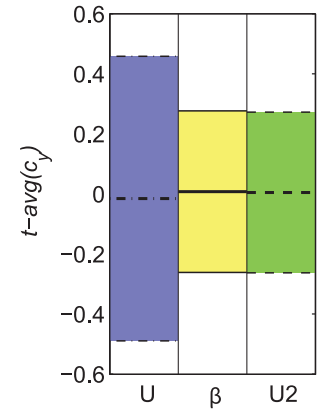

(b)

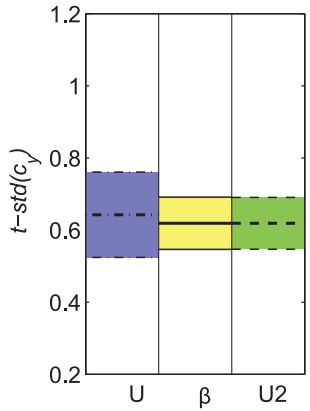

(c)

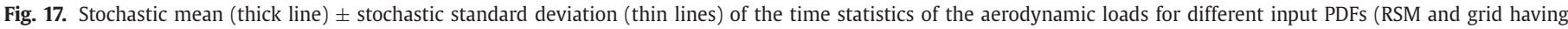

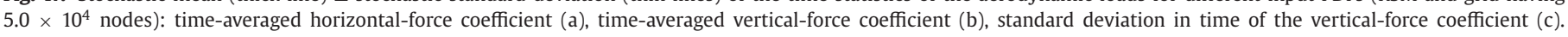

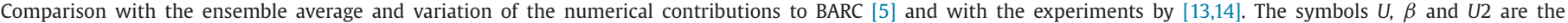
same as in Fig. 16.

and uniform distributions with the same input variance, thus the most important parameter seems to be the variance and not the shape of the input PDFs. The stochastic mean values of the time statistics of the aerodynamic loads obtained for the original uniform input PDF are very similar to those obtained for the other two input distributions, while the stochastic standard deviation is larger. As it was previously pointed out, the standard deviation of the initial uniform distribution is larger than the variance of the beta distribution on the same parametric interval.

The same holds for the statistics of the surface pressure coefficient (see Fig. 18). The major effect of changing the input probability distribution is observed in the stochastic standard deviation of the side - $\operatorname{avg}\left(t-\operatorname{std}\left(C_{p}\right)\right)$; as for the time statistics of the aerodynamic loads, the original uniform distribution leads to a larger output stochastic variance than the beta distribution, while the results obtained with the beta distribution are practically not distinguishable from those given by the uniform distribution having the same $\sigma$. Analogous considerations can also be made from the analysis of the UQ results for the pressure and velocity fields (not shown herein for the sake of brevity).

\section{Effect of the turbulence model on the UQ results}

In this Section the UQ results obtained by using the SST $k-\omega$ turbulence model are compared with the ones given by the RSM, in order to highlight the effect of the selection of the turbulence model on the propagation of aleatoric uncertainties. The same grid having $5 \times 10^{4}$ nodes is chosen, to single out the effect of turbulence modeling from that of discretization errors. The original uniform probability distribution of the input parameters is considered in both cases.

Differences in the stochastic mean values of the time statistics of the aerodynamic loads are found, especially for $t-\operatorname{avg}\left(c_{x}\right)$ and $t-\operatorname{std}\left(c_{y}\right)$ (see Fig. 19). Moreover, for all the considered quantities, the stochastic standard deviation is larger for the RSM compared to the SST $k-\omega$ turbulence model, meaning that this latter is less sensitive to uncertainties in the considered set-up parameters. For both models, however, the UQ dispersion for $t-\operatorname{avg}\left(c_{x}\right)$ and $t-\operatorname{std}\left(c_{y}\right)$ is significantly smaller than the one found in the BARC numerical contributions (see the previous discussion of the UQ results for RSM in Section 6.1). As previously observed, a large variability of $t-\operatorname{avg}\left(c_{y}\right)$ is observed, because of the considered variation in the angle of attack.
Table 7

Stochastic variance decomposition of the time statistics of the aerodynamic loads; SST $k-\omega$ and grid having $5.0 \times 10^{4}$ nodes.

\begin{tabular}{llllllll}
\hline & $S_{\alpha}$ & $S_{I}$ & $S_{L}$ & $S_{\alpha-I}$ & $S_{\alpha-L}$ & $S_{I-L}$ & $S_{\alpha-I-L}$ \\
\hline$t-\operatorname{avg}\left(c_{x}\right)$ & 0.880 & 0.101 & 0.005 & 0 & 0 & 0.014 & 0 \\
$t-\operatorname{avg}\left(c_{y}\right)$ & 1.000 & 0 & 0 & 0 & 0 & 0 & 0 \\
$t-\operatorname{std}\left(c_{y}\right)$ & 0.379 & 0.520 & 0.032 & 0.001 & 0 & 0.068 & 0
\end{tabular}

Regarding the pressure coefficient distribution, for both turbulence models the propagation of the uncertainty in the inlet conditions is considerably narrower than the overall dispersion of BARC numerical results (see Fig. 20). As observed for the aerodynamic loads, the stochastic standard deviations of the time-averaged and of the standard deviation in time of the pressure coefficient are definitely smaller for the SST $k-\omega$ than for the RSM. In particular, the maximum values of the stochastic standard deviation of $t-\operatorname{avg}\left(C_{p}\right)$ and $t-\operatorname{std}\left(C_{p}\right)$ for the SST $k-\omega$ model are 0.012 and 0.004 , to be compared with 0.041 and 0.046 obtained for the RSM (see Section 6.1). Significant differences between the results of the two turbulence models are found also in terms of stochastic mean of $t-\operatorname{std}\left(C_{p}\right)$ (see Fig. 20), much larger than the variability due to the considered uncertainties.

As previously highlighted, the mean and fluctuating pressure distribution on the cylinder side is strongly linked to the mean flow topology and, in particular, with the location of the mean flow reattachment. Fig. 21 shows the stochastic mean \pm standard deviation of the side-averaged reattachment point $x / D$ coordinate obtained with the SST $k-\omega$ model and RSM (already shown in Fig. 14) compared with the ensemble average and standard deviations of the numerical contributions reviewed in [5]. The stochastic mean values obtained with the SST $k-\omega$ model are comparable to the ensemble average of the BARC simulation results. Finally, also for this quantity, the stochastic variance of the predictions of the SST $k-\omega$ model is reduced compared to that of in RSM.

The uncertainty propagation results on the complete velocity and pressure field (not shown herein for the sake of brevity) showed that the distributions of stochastic mean and standard deviation are qualitatively similar for the two turbulence models. However, more quantitatively, the values of the stochastic standard deviation are lower for the SST $k-\omega$, as it was remarked previously.

Table 7 shows the partial variance decomposition of the time statistics of the aerodynamic loads for the SST $k-\omega$ model (to be 


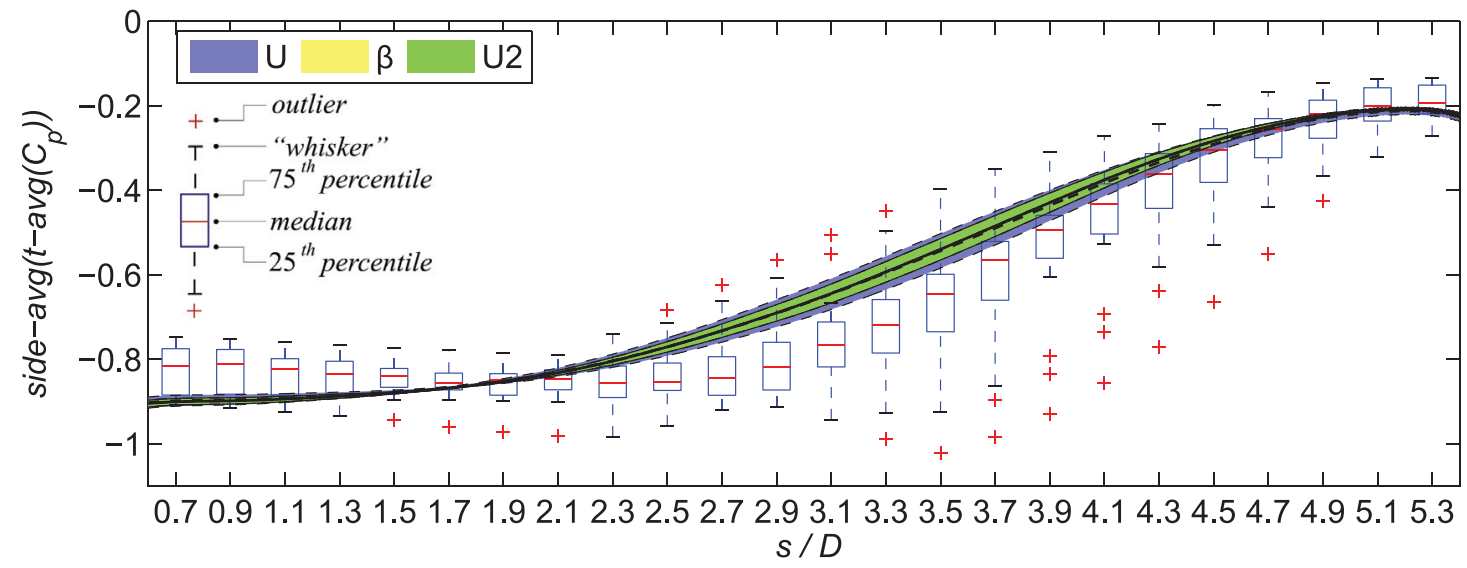

(a)

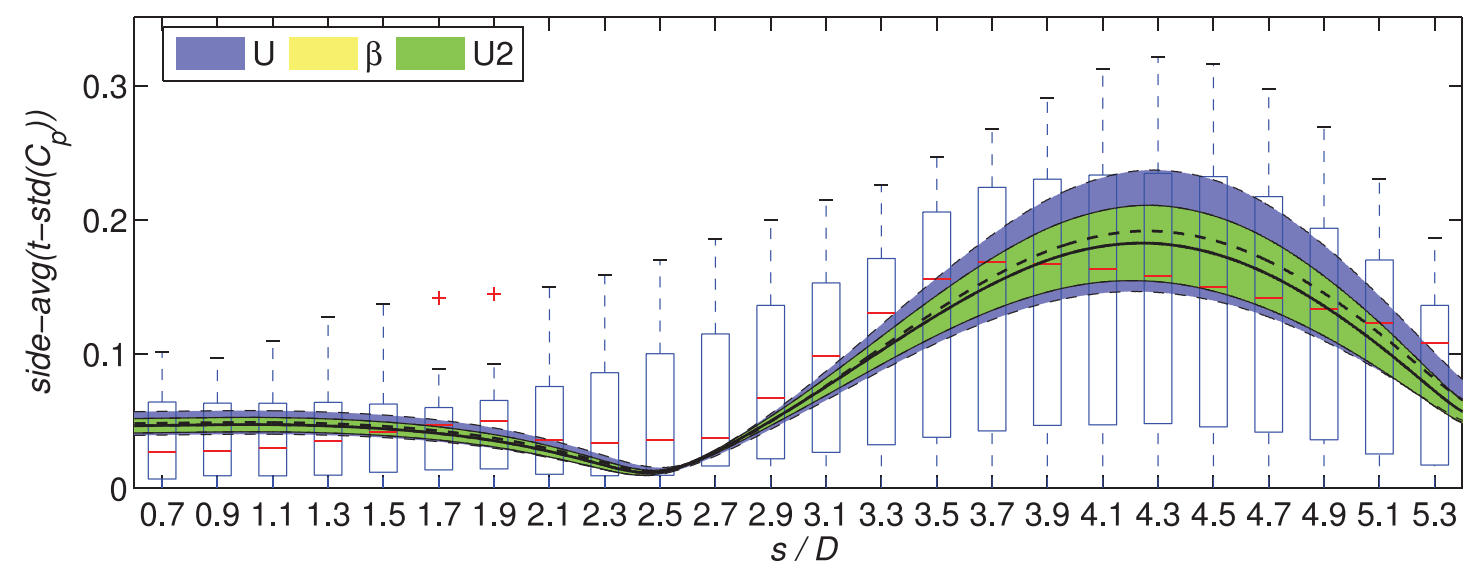

(b)

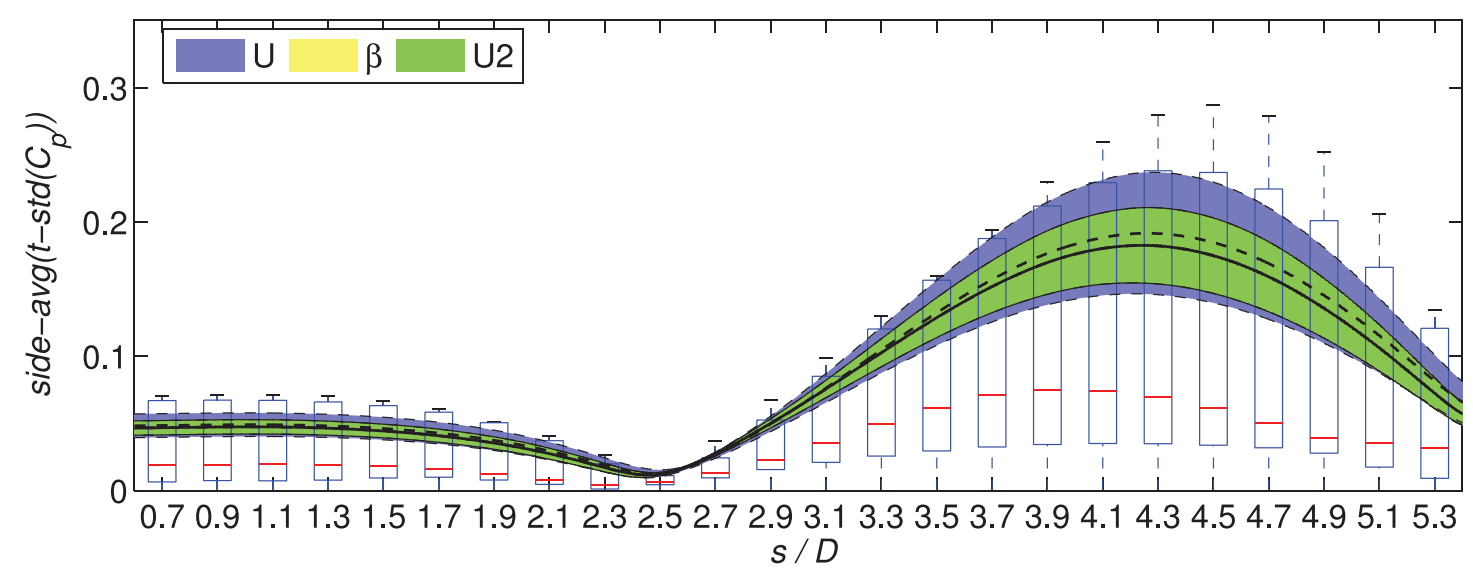

(c)

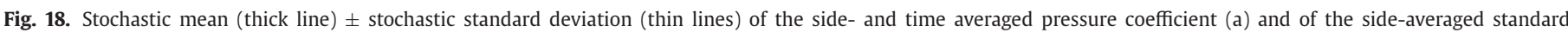

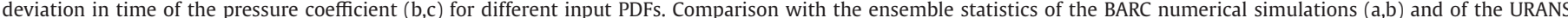

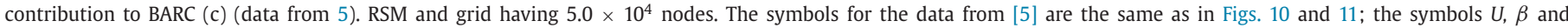
$U 2$ are the same as in Fig. 16. 


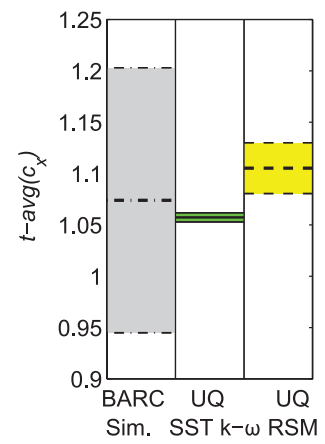

(a)

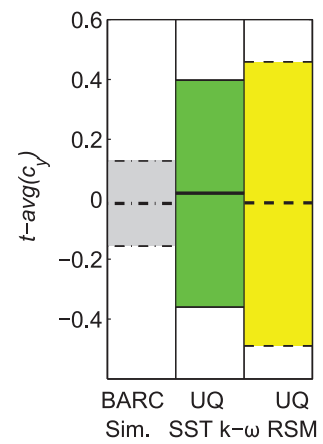

(b)

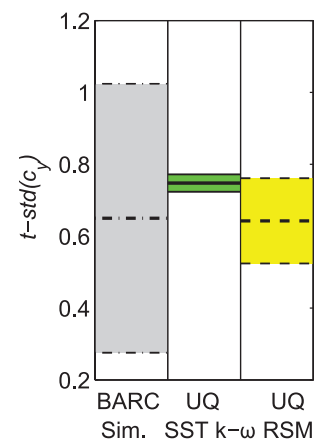

(c)

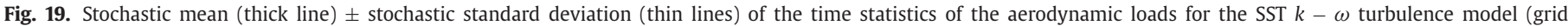

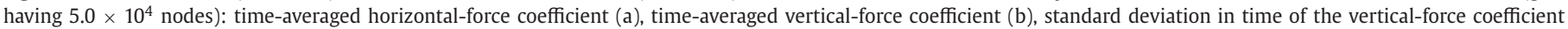
(c). Comparison with the results for the RSM on the same grid and with the ensemble average and variation of the numerical contributions to BARC (data from 5 ).

compared with Table 6). As expected, the variance of $t-\operatorname{avg}\left(c_{y}\right)$ is dominated by the uncertainty in the angle of attack. For $t-$ $\operatorname{avg}\left(c_{x}\right)$ and $t-\operatorname{std}\left(c_{y}\right)$, it is interesting to note that the impact of uncertainties in the inlet turbulence intensity is noticeably reduced for the SST $k-\omega$ model compared with that observed for the RSM model. This reduced sensitivity to $I$ may also explain the reduced dispersion of the results obtained with the SST $k-\omega$ model: since with this model the sensitivity to $I$ is reduced and, as observed previously, $\alpha$ and $L$ are less impacting parameters, this leads to a smaller variances in the quantities of interest.

The partial stochastic variances of the time-averaged and standard deviation of the surface pressure coefficient, due to the single set-up parameters, are shown in Fig. 22. Compared to the analogous partial variances for the RSM, shown in Fig. 15 and commented in Section 6, the sensitivity to the freestream turbulence intensity is once again noticeably lower for the SST $k-\omega$ model. As observed previously for the aerodynamic loads, this may explain the reduced global stochastic variance of the time-averaged and fluctuating pressure distribution over the cylinder side observed for the SST $k-\omega$ model

\section{Effect of grid resolution on the UQ results}

In this Section the effects of the spatial discretization error on the UQ results are analyzed, by comparing the UQ results obtained by using both turbulence models on the grid having $5 \times 10^{4}$ nodes with the ones obtained on the grid having $1.2 \times 10^{4}$ nodes.

The main effect of grid resolution is on the stochastic mean value of the time statistics of the aerodynamic loads (see Fig. 23), while the stochastic variances remain relatively unchanged. The same observation can be made for the time average and standard deviation of the pressure coefficient on the cylinder side (see Fig. 24) and for the reattachment point location on the cylinder side (Fig. 25).

Thus, it seems that the discretization error does not significantly affect the propagation of aleatoric uncertainties for both the turbulence models selected in this study.

\section{Concluding remarks}

The propagation of aleatoric uncertainties in three freestream flow parameters of the BARC configuration, namely the angle of attack, the freestream turbulence intensity and length scale, was investigated through two-dimensional URANS simulations and a stochastic collocation method. The effect of the chosen turbulence model was appraised by repeating the stochastic analysis for two different models, namely SST $k-\omega$ and RSM, on the same grid. Furthermore, the impact on the UQ results of the assumed PDF shape for the input uncertain parameters was quantified for the RSM. Finally, the impact of spatial discretization was also estimated by carrying out the UQ and sensitivity analyses for two different grid resolutions.

The quantities of interest that have been found to be the most sensitive to the considered uncertainties are those that are also characterized by the largest dispersion among the BARC contributions, as e.g. the standard deviations in time of the vertical-force coefficient and of the pressure distribution over the cylinder surface. Nonetheless, the propagation of the considered set-up uncertainties leads to a variability of the present results which is smaller than that of the BARC experimental contributions. Thus, it seems that the dispersion of the experimental data collected in BARC can not be solely explained by the considered uncertainties in the freestream flow conditions.

A practical issue in stochastic sensitivity analysis is the lack of information on the PDFs of the uncertain input parameters, which are usually guessed. Hence, it is interesting to evaluate how much this guess impacts on the UQ results. In our case, it has been found that the most important parameter is the variance and not the shape of the input PDFs and that this affects the stochastic standard deviation of the quantities of interest, while the stochastic mean values remain practically unchanged.

Another issue is how much the sensitivity to the uncertainties in the freestream parameters obtained in the present work depends on the turbulence modeling. Indeed, it has been found that the turbulence model has a significant effect on the statistic mean but also on the the variability of the output quantities with the set-up parameter uncertainties. In particular, the stochastic standard deviations for all the time statistics of the aerodynamic loads and for the pressure coefficient distributions are larger for the RSM than for the SST $k-\omega$ turbulence model. Considering the partial variances to each input parameter, the results obtained with the RSM model are significantly more sensitive to the uncertainty in the freestream turbulence intensity than those of the SST $k-\omega$ model. This may also explain the larger variability of the results given by RSM. For both turbulence models, the freestream turbulence length scale $L$ is the less influential parameter. One may wonder whether in a simulation in which part of the turbulence scales are resolved, as in LES, the sensitivity to the freestream tur- 


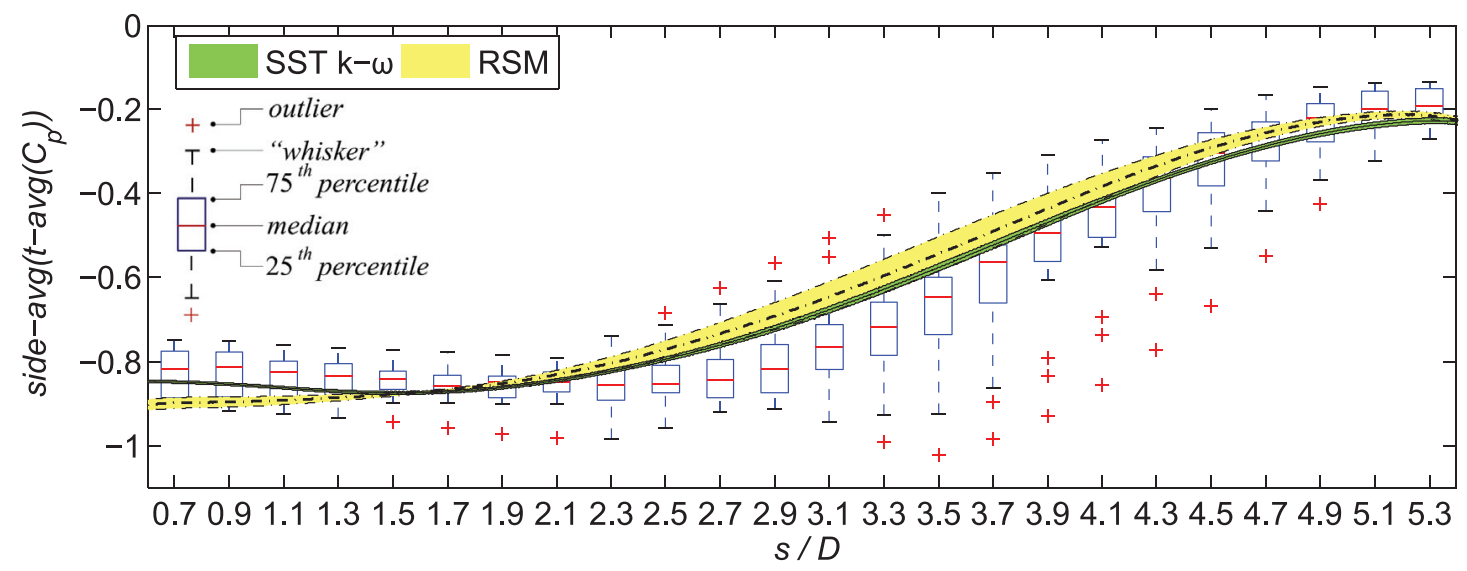

(a)

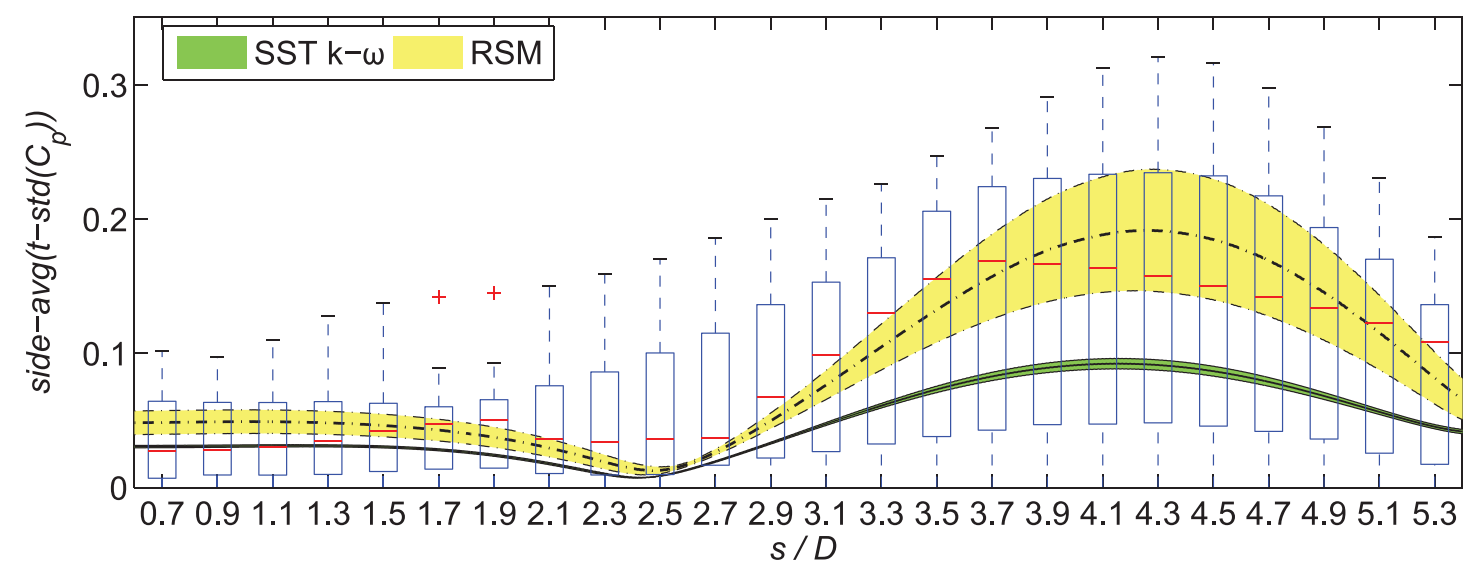

(b)

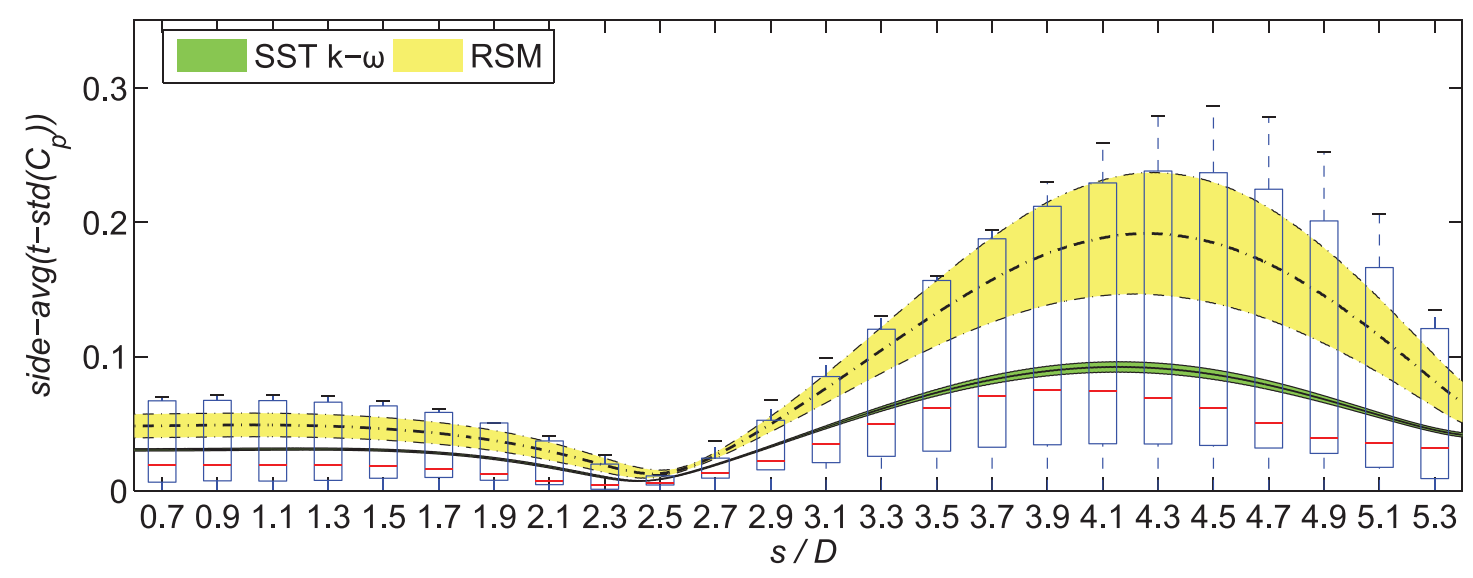

(c)

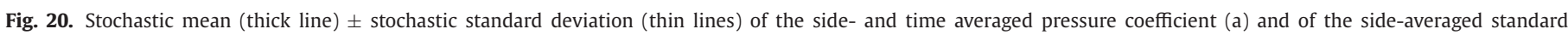

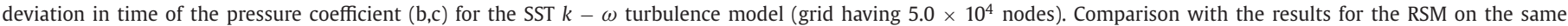

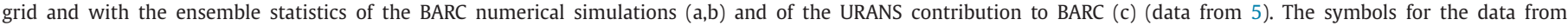
[5] are the same as in Figs. 10 and 11. 


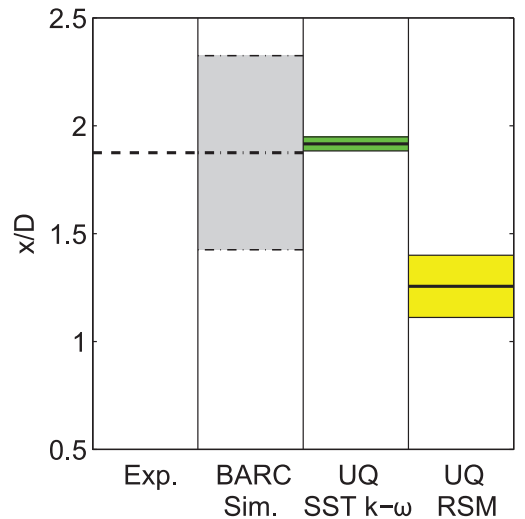

Fig. 21. Stochastic mean (thick line) \pm stochastic standard deviation (thin lines) of the side-averaged streamwise coordinate of the mean flow reattachment point for the SST $k-\omega$ turbulence model (grid having $5.0 \times 10^{4}$ nodes). Comparison with the results for the RSM on the same grid and with the ensemble statistics of the BARC numerical simulations and with the experimental data (data from 5).

bulence features would be larger. This issue would require further investigation, but this implies remarkable additional difficulties. In- deed, in addition to the large computational costs of each simulation, the problem of generating suitable freestream conditions for LES when only bulk information on the turbulence features is available, as e.g. turbulence intensity and scale, is still an open issue in the literature. Therefore, another source of uncertainty/error related to the used methodology for generation of freestream conditions would be introduced.

Furthermore, the discretization error has been estimated herein by considering UQ results on two different grids. The main effect of grid resolution is a small variation of the stochastic mean value of the quantities of interest, while the stochastic standard deviations are almost unchanged. Thus, it seems that the discretization errors do not significantly affect the propagation of the considered aleatoric uncertainties in the freestream parameters, at least for the turbulence models selected in this study.

Additional aleatoric uncertainties are present in the experiments; the main ones are related to the model geometry, and, in particular, to the sharpness of the corners and to the surface roughness. The stochastic methodology used in the present analysis can in principle be adopted also to quantify the impact of these geometrical uncertainties, although additional technical issues are present in this case, such as defining stochastic input distributions

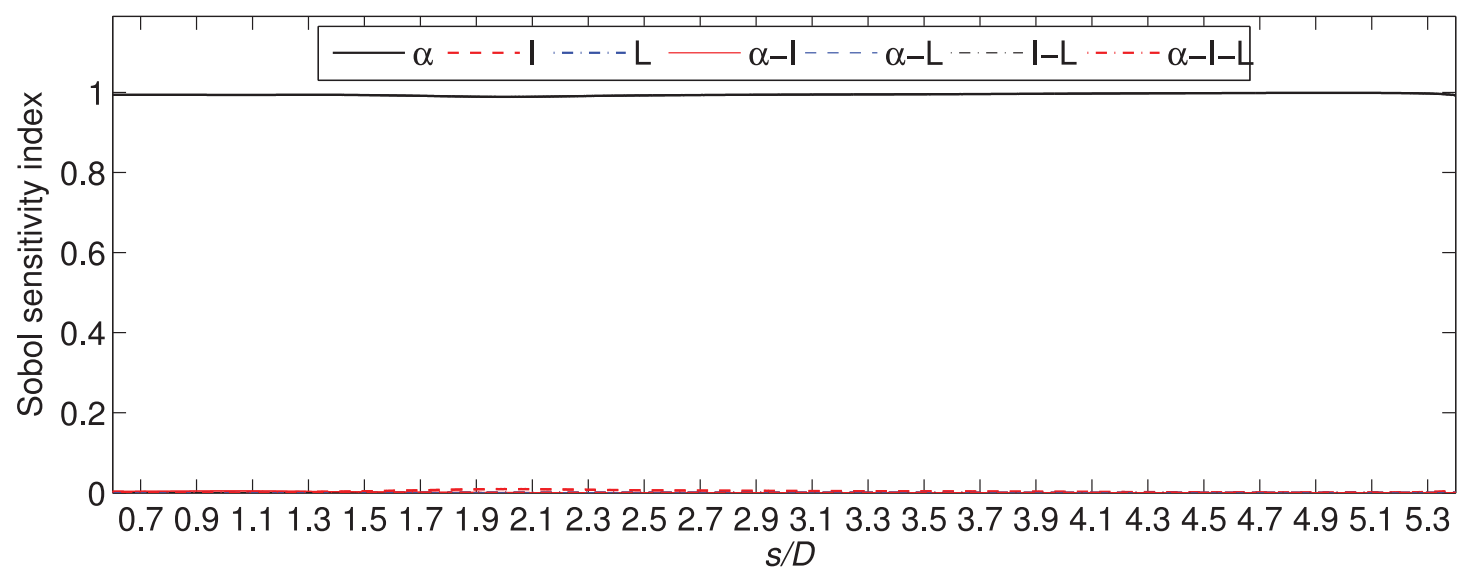

(a)

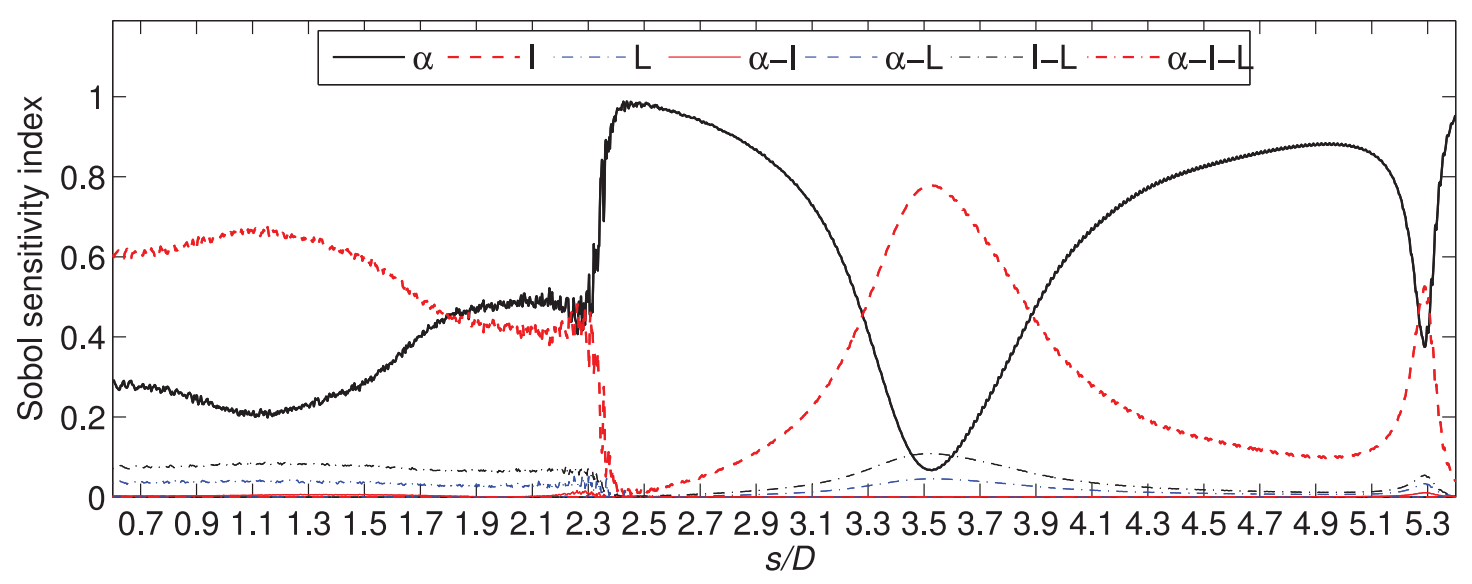

(b)

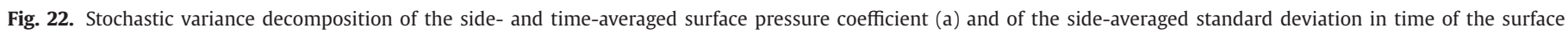
pressure coefficient (b) (for the SST $k-\omega$ model and grid having $5.0 \times 10^{4}$ nodes). 


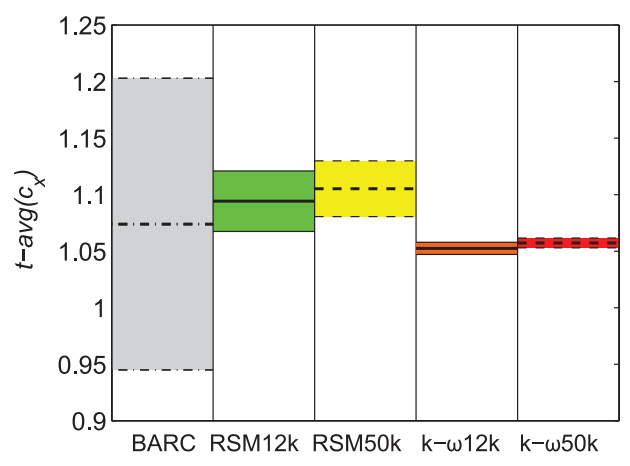

(a)

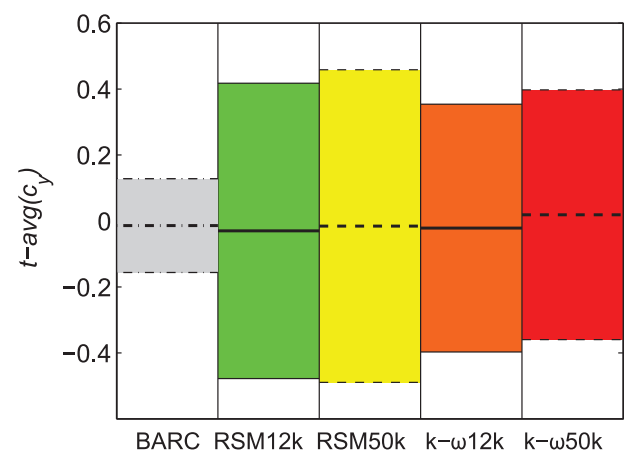

(b)

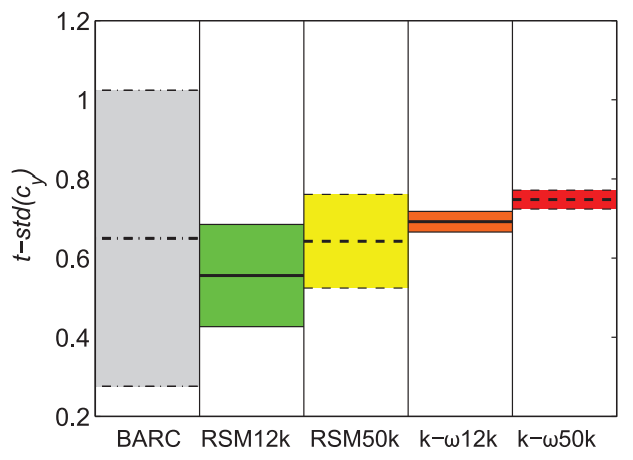

(c)

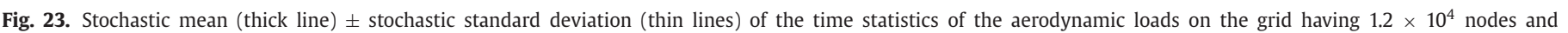

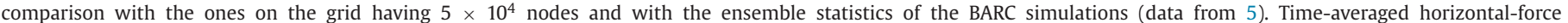
coefficient (a), time-averaged vertical-force coefficient (b) and standard deviation in time of the vertical-force coefficient (c).

of spatially varying geometrical uncertainties and the need for remeshing.

Other known differences exist in the set-up of the BARC experiments, namely different blockage ratios and different spanwise lengths of the model. The differences in blockage ratio, in the range of the BARC experiments, are not expected to have a significant impact, as confirmed by a deterministic numerical sensitivity analysis carried out herein. Conversely, the differences in the spanwise length of the model might be important. As previously discussed, however, a numerical stochastic analysis of the impact of the spanwise length would imply huge computational costs. Indeed, it is questionable whether a URANS approach can accurately capture 3D effects. Large-eddy simulation would be more suitable, but that type of approach requires very fine grid resolution, which increases dramatically the computational cost of each deterministic simulation. This is beyond the scope of the present paper and could be the object of future research.
Finally, from a practical point of view, the results of the present work give useful clues for URANS simulations of the BARC configuration and of similar problems. For instance, it appears that it is not important to reproduce the freestream turbulence length, which is usually not available from experiments.

\section{Acknowledgements}

The authors are grateful to Luca Bruno for the interesting discussions which stimulated the present study and for his precious suggestions. The authors wish also to thank Francesco Ascenso for his contribution in carrying out the numerical simulations and Stijn Desmedt for implementing the code for Sobol variance calculations. 


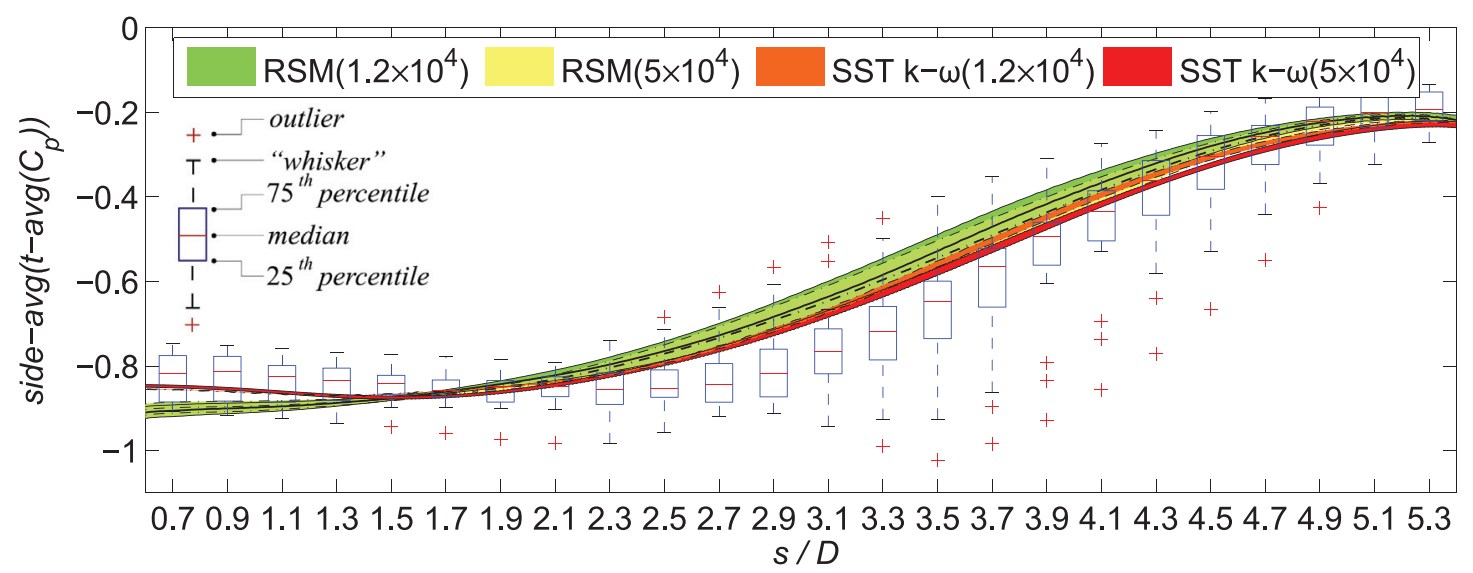

(a)

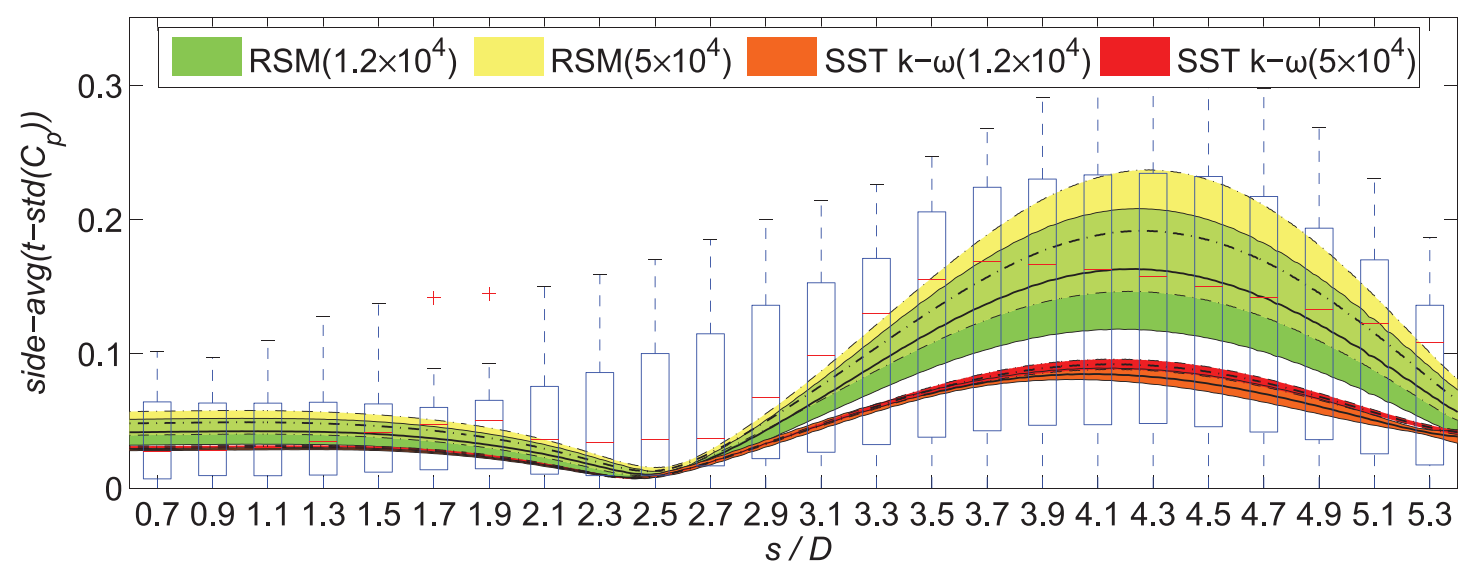

(b)

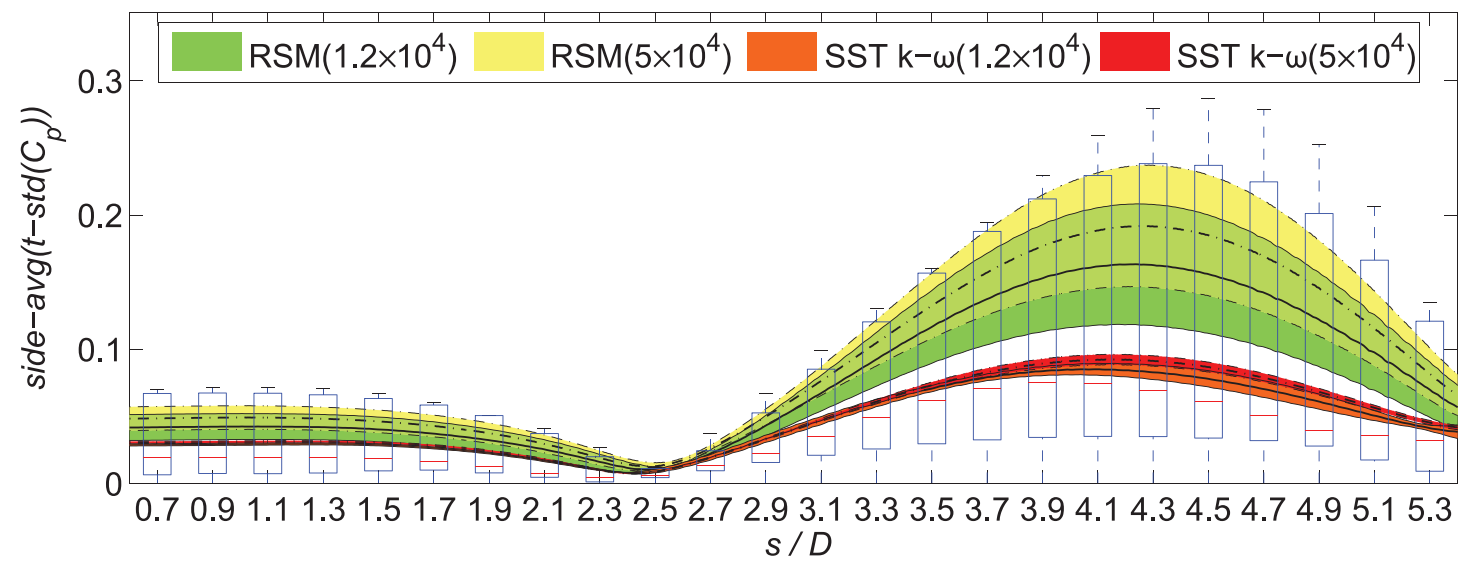

(c)

Fig. 24. Stochastic mean (thick line) \pm stochastic standard deviation (thin lines) of the side- and time averaged pressure coefficient (a) and of the side-averaged standard deviation in time of the pressure coefficient $(b, c)$ for different grid resolutions and turbulence models (grid having $5.0 \times 10^{4}$ nodes). Comparison with the ensemble statistics of the BARC numerical simulations (a,b) and of the URANS contribution to BARC (c) (data from 5). The symbols for the data from [5] are the same as in Figs. 10 and 11. 


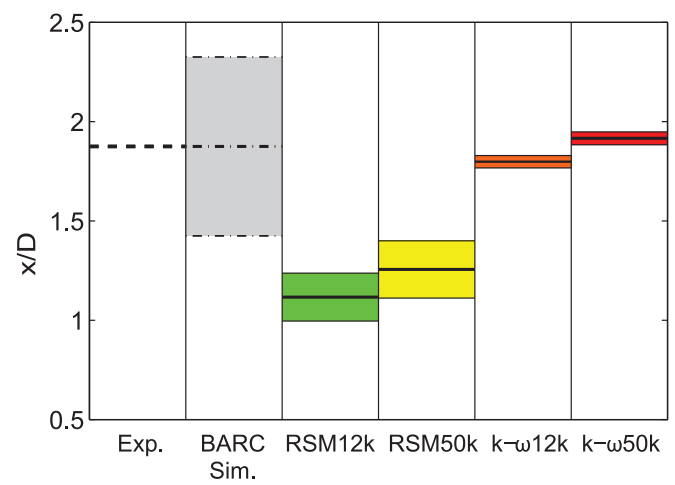

Fig. 25. Stochastic mean (thick line) \pm stochastic standard deviation (thin lines) of the side-averaged streamwise coordinate of the mean flow reattachment point for different grid resolutions and turbulence models. The results are also compared with the ensemble statistics of the BARC numerical simulations and with the experimental data (data from 5).

\section{References}

[1] ANSYS Fluent. Help system, Fluent 6.3 user's guide. ANSYS, Inc 2006.

[2] Bartoli G., Bruno L., Buresti G., Ricciarelli F., Salvetti M. V., Zasso A. BARC overview document: requests for computational simulations and requests for wind tunnel tests. http://wwwaniv-iaweorg/barc. 2008.

[3] Bruno L, Fransos D. Probabilistic evaluation of the aerodynamic properties of a bridge deck. J Wind Eng Ind Aerod 2011;99:718-28.

[4] Bruno L, Fransos D, Coste N, Bosco A. 3D flow around a rectangular cylinder: a computational study. J Wind Eng Ind Aerod 2010;98:263276.
[5] Bruno L, Salvetti MV, Ricciarelli F. Benchmark on the Aerodynamics of a Rectangular 5:1 Cylinder: an overview after the first four years of activity. J Wind Eng Ind Aerod 2014;126:87-106.

[6] Desmedt S. Dimension-adaptive sparse grid for industrial applications using Sobol variances. TU Delft, Delft University of Technology; 2015. Ph.D. thesis.

[7] Grozescu AN, Bruno L, Fransos D, Salvetti MV. Large-eddy simulations of a Benchmark on the Aerodynamics of a Rectangular 5:1 Cylinder. Proceedings of the 20th Italian conference on theoretical and applied mechanics, Bologna, Italy 2011.

[8] Issa RI. Solution of the implicit discretized fluid flow equations by operator splitting. J Comput Phys 1986;65 (1):40-65.

[9] Launder BE, Reece GJ, Rodi W. Progress in the development of a Reynolds-stress turbulence closure. J Fluid Mech 1975;68:537-66.

[10] Mannini C, Šoda A, Schewe G. Unsteady RANS modelling of flow past a rectangular cylinder: Investigation of Reynolds number effects. Comp Fluids 2010;39 (9):1609-24.

[11] Menter FR. Two-equation eddy-viscosity turbulence models for engineering applications. AIAA J 1994;32(8):1598-605.

[12] Oberkampf WL, Roy CJ. Verification and validation in scientific computing. 1st ed. New York, NY, USA: Cambridge University Press; 2010.

[13] Schewe G. Influence of the Reynolds-number on flow-induced vibrations of generic bridge sections. In: Radi J, editor. proceedings of the international conference on bridges, Dubrovnik, Croatia; 2006. p. 351-8.

[14] Schewe G. Reynolds-number-effects in flow around a rectangular cylinder with aspect ratio 1:5. In: Proceedings of the fifth European and African conference on wind engineering, Florence, Italy; 2009.

[15] Shoeibi Omrani P, O'Mahoney T, Mack A, Witteveen J. Uncertainty quantification of heavy gas release over a barrier. In: Proceedings of the 12th international conference on applications of statistics and probability in civil engineering, Vancouver, Canada; 2015.

[16] Smolyak SA. Quadrature and interpolation formulas for tensor products of certain classes of functions. Dokl Akad Nauk SSSR 1963;4:240-3.

[17] Sobol IM. Global sensitivity indices for nonlinear mathematical models and their Monte Carlo estimates. Math Comput Simul 2001;55(1):271-80.

[18] Tang G, Iaccarino G, Eldred M. Global sensitivity analysis for stochastic collocation. AIAA-2010-2589 2010.

[19] Xiu D, Hesthaven JS. High-order collocation methods for differential equations with random inputs. SIAM J Sci Comput 2005;27:1118-39. 